Draft VERSion July 14, 2020

Typeset using $\mathrm{LAT}_{\mathrm{E}} \mathrm{X}$ twocolumn style in AASTeX63

\title{
Bar Classification based on the Potential Map
}

\author{
Yun Hee Lee, ${ }^{1}$ Myeong-Gu Park, ${ }^{1,2}$ Hong Bae Ann, ${ }^{3}$ Taehyun Kim, ${ }^{1}$ and Woo-Young SeO ${ }^{4}$ \\ ${ }^{1}$ Department of Astronomy and Atmospheric sciences, Kyungpook National University, Daegu, 41566, Republic of Korea \\ ${ }^{2}$ Research and Training Team for Future Creative Astrophysicists and Cosmologists (BK21 Plus Program), Kyungpook National \\ University, Daegu, 41566, Republic of Korea \\ ${ }^{3}$ Department of Earth Science Education, Pusan National University, Busan, 46241, Republic of Korea \\ ${ }^{4}$ Department of Physics, Chungbuk National University, Cheongju, 28644, Republic of Korea
}

(Received March 16, 2020; Revised June 15, 2020; Accepted July 8, 2020)

Submitted to ApJ

\begin{abstract}
We introduce a new approach to classify barred galaxies that utilizes the transverse-to-radial force ratio map (ratio map, hereafter) in a different manner from previous studies. When we display the ratio map in polar coordinates, barred galaxies appear as four aligned, horizontal thick slabs. This characteristic feature enables us to successfully classify barred and nonbarred galaxies with an accuracy of $87 \%$. It yields the bar fraction of $53 \%$, including both SBs and SABs, when applied to 884 nearby $(z<0.01)$ spiral galaxies from the Sloan Digital Sky Survey/DR7. It also provides the bar strength and length measurements, in particular, separated from the spiral arms. They show good correlations with the measures estimated from ellipse fitting and Fourier analysis. However, we find different tendencies of the bar strength measurements in terms of the Hubble sequence: as the Hubble sequence increases (towards late-type), the bar strength and bar ellipticity increase, whereas the dipole Fourier amplitude decreases. We show that the bulge affects the estimation of the bar strength differently, depending on the classification methods. The bulge causes the bar length to be overestimated in all three methods. Meanwhile, we find that barred galaxies show two types of radial profiles of the angle-averaged force ratio: one has a maximum peak (type $\mathrm{M}$ ) and the other a plateau (type $\mathrm{P}$ ). Comparison with numerical simulations suggests that type $\mathrm{M}$ bars are more mature than type $\mathrm{P}$ bars in terms of evolutionary stage.
\end{abstract}

Keywords: galaxies: evolution - galaxies: formation - galaxies: classification - galaxies: spiral galaxies: structure

\section{INTRODUCTION}

Roughly $60 \%$ of disk galaxies in the local universe have bars (de Vaucouleurs et al. 1991; Buta et al. 2010a, 2015; Ann et al. 2015). Bars play significant roles in the secular evolution of galaxies: bars drive gas and stars into the center of galaxies (Roberts et al. 1979; van Albada, \& Roberts 1981; Schwarz 1981, 1984; Prendergast 1983; Combes, \& Gerin 1985; Athanassoula 1992; Sellwood, \& Wilkinson 1993; Ann, \& Thakur 2005), increase the central star formation (Heckman 1980; Martin 1995; Huang et al.

Corresponding author: Myeong-Gu Park

mgp@knu.ac.kr
1996; Ellison et al. 2011; Oh et al. 2012), global star formation (Martinet \& Friedli 1997; Aguerri 1999), and central mass concentration (Pfenniger \& Norman 1990; Ann \& Lee 2000; Athanassoula 2002), and build up bulge-like structures such as pseudobulges and peanut-shaped bulges (Kormendy 1982; Pfenniger \& Norman 1990; Kormendy \& Kennicutt 2004; Yoshino, \& Yamauchi 2015; Li, \& Shen 2015; Li et al. 2017).

In addition, bars are deeply associated with substructures such as spiral arms, rings, and central dust structures. As bars become stronger, spiral arms (Buta et al. 2009; Salo et al. 2010) and rings (Grouchy et al. 2010) become stronger, pitch angles (Font et al. 2019) and rings (Ann 2001; Knapen et al. 2002; Comerón et al. 2010; Kim et al. 2012) smaller, and dust lanes straighter 
(Athanassoula 1992; Knapen et al. 2002; Comerón et al. 2009; Kim et al. 2012; Sánchez-Menguiano et al. 2015). Athanassoula et al. (2009a,b, 2010) explained that the bar strength determines ring and spiral morphologies based on the orbital motion driven by the unstable equilibrium points in the bar potential (Romero-Gómez et al. 2006, 2007). As such, bar strength is an important parameter that characterizes the bars, along with bar length and pattern speed (Aguerri et al. 2015; Sánchez-Menguiano et al. 2015).

We generally measure the bar strength by the bar ellipticity (Martinet \& Friedli 1997; Aguerri 1999; Abraham \& Merrifield 2000; Laurikainen et al. 2002; Marinova \& Jogee 2007; Aguerri et al. 2009), the normalized Fourier amplitude (Ohta et al. 1990; Aguerri et al. 2000; Athanassoula \& Misiriotis 2002), or the maximum transverse-to-radial force ratio $Q_{\mathrm{b}} \quad$ (Combes \& Sanders 1981; Buta \& Block 2001; Laurikainen \& Salo 2002; Buta et al. 2004; Díaz-García et al. 2016). They show comparatively good correlations among each other, although scatters are not small (Block et al. 2001; Laurikainen et al. 2002; Laurikainen \& Salo 2002; Díaz-García et al. 2016).

The force ratio is the representative of the physical bar strength based on the potential calculation. We expect that the force reflects the physical nature of the bar better than the light distribution. This photometric force ratio is tightly correlated with the kinematic bar strength based on the stellar velocity field (Seidel et al. 2015). It is useful when comparing the theory against observational results as well (Athanassoula et al. 2009a,b, 2010).

In this paper, we investigate not only the force ratio but also the patterns on the transverse-to-radial force ratio map (hereafter, the ratio map). Sanders \& Tubbs (1980) and Combes \& Sanders (1981) first proposed a measure of the bar strength by the maximum ratio of the tangential force to the mean radial force $Q_{\mathrm{b}}$. Regan \& Teuben (2004) argued that $Q_{\mathrm{b}}$ alone is not sufficient to determine the bar orbit families and their morphologies, and, therefore, the net mass inflow into the nuclear region. Since $Q_{\mathrm{b}}$ is a single global parameter, it may not be able to account for every bar-related process as well as we would like. But, we find that $Q_{\mathrm{b}}$ is correlated with the central mass inflow at least better than the quadrupole moment and at a similar significance as the bar axis ratio when we reinvestigate the simulation results displayed in their Figures 1 - 6 .

Quillen et al. (1994) developed a procedure to calculate the gravitational potential map by solving the Poisson equation using the fast Fourier transform (FFT). Buta \& Block (2001, hereafter BB01) applied this pro- cedure to some number of galaxies, found a butterfly pattern on the ratio map, and proposed it as the bar signature. However, there has been little further effort to investigate or utilize this pattern. This contrasts with many studies to refine the estimation of the force ratio by taking into account the dependence of the vertical scaleheight on the Hubble type (Laurikainen et al. 2002; Laurikainen \& Salo 2002), the gradient of the vertical scaleheight as a function of distance (Laurikainen \& Salo 2002; Díaz-García et al. 2016), the bulge stretching (Díaz-García et al. 2016), and the dark matter halo (Buta et al. 2004; Díaz-García et al. 2016). Here, we simplify and analyze the patterns on the ratio map by converting it from the Cartesian coordinates to the polar coordinates. It enables us to explore a new automated classification for barred galaxies.

Among the three methods to measure the bar strength, the ellipse fitting and Fourier analysis have been widely utilized to automatically detect barred galaxies (Ohta et al. 1990; Wozniak et al. 1995; Aguerri et al. 2000, 2009; Laurikainen \& Salo 2002; Laurikainen et al. 2004a; Jogee et al. 2004; Marinova \& Jogee 2007; Barazza et al. 2008; Marinova et al. 2009, 2010, 2012; Consolandi 2016). They have been the most representative ways for bar classification and show good agreements for SA and SB galaxies classified by visual inspection at $70 \%$ to $85 \%$ level, respectively (Lee et al. 2019, hereafter Paper I). However, in Paper I, we found that the ellipse fitting method often misses bars, in particular, early-type spirals with a large bulge, and the Fourier analysis usually detects only strongly barred galaxies. This causes contradictory dependences of bar fraction on the Hubble type, depending on whether the ellipse fitting method or Fourier analysis is applied, even when applied to the same sample of galaxies.

Recent studies of barred galaxies have utilized visual inspections (Nair \& Abraham 2010; Oh et al. 2012; Lee et al. 2012; Buta et al. 2015; Ann et al. 2015), especially owing to the availability of a visual classification by numerous anonymous citizens such as the Galaxy Zoo project (Masters et al. 2011; Hoyle et al. 2011; Skibba et al. 2012; Cheung et al. 2013; Simmons et al. 2014). However, the classification by a large number of amateurs can only deal with obviously, i.e., strongly, barred galaxies. Besides, visual classification cannot provide other characteristics for bars except the bar length. On the other hand, our new automated method provides a reliable classification and evaluations of the bar strength and bar length at the same time.

This paper is organized as follows. In Section 2, we describe our sample, data reduction, and the method to 
calculate the gravitational potential. In section 3, we show the ratio map in the polar coordinates and test the ratio map analysis on mock galaxies. We describe the automated classification method by analyzing the pattern of the ratio map in Section 4. In Section 5, we compare the bar strengths and lengths obtained by all three methods: the ellipse fitting method, Fourier analysis, and the potential method. Discussion and summary are given in Section 6 and Section 7 .

\section{DATA REDUCTION AND CALCULATION}

\subsection{Sample and Data Reduction}

The data are from Paper I, which consists of a volumelimited sample with 884 spiral galaxies from the Sloan Digital Sky Survey/DR7 that are brighter than $M_{\mathrm{r}}=$ -15.2 mag. Galaxies with $i>60^{\circ}$ and with frames smaller than $R_{25}$ were excluded. The sample originated from 1876 spiral galaxies with $z<0.01$ in the cata$\log$ of Ann et al. (2015, hereafter Ann15). This catalog provides a detailed classification of galaxies in terms of $\mathrm{SA}, \mathrm{SAB}$, and SB and of ten revised Hubble stages from $\mathrm{S} 0 / \mathrm{a}$ to $\mathrm{Sm}$ based on the visual inspection. This is one of the largest sample of barred galaxies which are further classified into SABs or SBs.

The near-infrared wavelength traces the dynamical potential best, and is far less affected by dust obscuration (Benedict et al. 1992; Quillen et al. 1994; Buta \& Block 2001; Laurikainen \& Salo 2002). For this reason, we used $i$-band images that are designed for the near-infrared wavelength in SDSS. All images have been reduced by subtracting the soft-bias and sky background gradient with a first-order polynomial fit. Bright clumpy sources such as foreground stars, adjacent galaxies, and star-forming regions in spiral arms have been masked with Gaussian model images, and all images have been smoothed with a box size of $0.1 R_{25}$ to reduce the artificial residuals after masking (Paper I).

We calculated the position angle and ellipticity at $R_{25}$ by fitting ellipses to isophotes following the method by Davis et al. (1985) and Athanassoula et al. (1990) and deprojected all galaxies assuming that the disk is a perfect circle at $R_{25}$. These processes are essential in reducing the uncertainty of bar strength that is easily affected by the orientation (BB01). We determined $R_{25}$ at the isophote of $25 \mathrm{mag} \operatorname{arcsec}^{-2}$ from the surface brightness profile in the $g$-band of the SDSS using the relation $m_{B}=m_{g}+0.3$ (Rodgers et al. 2006; Lee et al. 2019).

\subsection{Calculation of the Gravitational potential}

To calculate the gravitational potential, we adopt an assumption that the mass-to-light ratio is constant throughout the disk (Quillen et al. 1994). This assump- tion is supported by two kinds of observations: the dark matter is only a small fraction of the visible matter within $R_{25}$ (Kalnajs 1983; Kuijken \& Gilmore 1989; Flynn \& Fuchs 1994); the near-infrared colors are constant across the bar region with the typical old stellar population dominating the light (Frogel et al. 1978; Frogel 1985; Terndrup et al. 1994; Quillen et al. 1994). However, we need to be cautious that the mass can be overestimated at the nuclear region because it appears redder than the bar region (Quillen et al. 1994).

We calculated the gravitational potentials following the procedures of Quillen et al. (1994) and BB01. The potentials are calculated from the convolution of the image intensities with the vertical density profile of the disk. First, we expanded $i$-band deprojected images with four times the area in order to simulate an isolated system (Hohl 1972; Quillen et al. 1994). We solved for the gravitational potential from the Poisson equation by convolving the three-dimensional mass density $\rho(\mathbf{x})$ with $1 /\left|\mathbf{x}-\mathbf{x}^{\prime}\right|$

$$
\Psi(\mathbf{x})=-G \int \frac{\rho\left(\mathbf{x}^{\prime}\right) d^{3} \mathbf{x}^{\prime}}{\left|\mathbf{x}-\mathbf{x}^{\prime}\right|}
$$

using the FFT on Cartesian coordinates (Hohl \& Hockney 1969; Quillen et al. 1994; Buta \& Block 2001). We assumed that the vertical density distribution follows the exponential model,

$$
\rho_{z}(z)=\frac{1}{2 h_{z}} \exp \left(-\left|\frac{z}{h_{z}}\right|\right)
$$

with the scaleheight $h_{z}$ (Elmegreen \& Block 1999; Laurikainen \& Salo 2002; Buta et al. 2004). In equation $1, \rho(\mathbf{x})$ can be written as $\rho(\mathbf{x})=\sum(x, y) \rho_{z}(z)$, where $\sum(x, y)$ is the mass surface density in the plane of the galaxy and $\rho_{z}(z)$ is normalized as $\int_{-\infty}^{\infty} \rho(z) d z=1$. The gravitational potential at the central plane of the galaxy can be described as

$\left.\Psi(x, y, z=0)=-G \int_{-\infty}^{\infty} \int_{-\infty}^{\infty} \sum\left(x^{\prime}, y^{\prime}\right) g\left(x-x^{\prime}, y-y^{\prime}\right) d x^{\prime} d\left(y^{\prime}\right)^{\prime}\right)$

where $g\left(x-x^{\prime}, y-y^{\prime}\right) \equiv g(r)$ defined as

$$
g(r)=\int_{-\infty}^{\infty} \frac{\rho_{z}(z)}{\sqrt{r^{2}+z^{2}}} d z
$$

with

$$
r^{2}=\left(x-x^{\prime}\right)^{2}+\left(y-y^{\prime}\right)^{2}
$$

(Quillen et al. 1994; Laurikainen \& Salo 2002).

When we estimated the scaleheight $h_{z}$, we took into account the different ratio of disk scalelength to vertical scaleheight $h_{r} / h_{z}$ for different Hubble types $T: 4$ 
for $T \leq 1,5$ for $2 \leq T \leq 4$, and 9 for $T \geq 5$ (de Grijs 1998; Laurikainen et al. 2004b; Díaz-García et al. 2016). We measured the disk scalelength $h_{r}$ in the exponential fit, $I(r)=I_{0} \exp \left(-r / h_{r}\right)$, from the surface brightness profile obtained from the ellipse fitting. When the extrapolated profile into the bulge region deviates from the observational profile, however, we adopt an inner-truncated profile, $I(r)=I_{0} \exp \left[-\left(\alpha r+\beta^{\mathrm{n}} / r^{\mathrm{n}}\right)\right]$ (Kormendy 1977). Kormendy (1977) explained that the sharp transition occurs because stars in the disk move in nearly circular orbits while those in the bulge have an isotropic velocity distribution. Dust extinction or the bar existence have also been suggested as the possibilities for the origin of the innertruncation (Baggett et al. 1998; MacArthur et al. 2003; Anderson et al. 2004; Foyle et al. 2008; Puglielli et al. 2010). We measure the disk scalelength $h_{r}$ by $\alpha^{-1}$. The parameter $n$ determines the sharpness of the cutoff and the specific radius $\beta$ represents a sharp inner edge.

There can be other choices in the calculation of the potential. It can be evaluated on a polar coordinate grid instead of on a Cartesian grid (Salo et al. 1999; Laurikainen \& Salo 2002; Buta et al. 2004). The isothermal density function can be chosen as well to model the vertical profile of the disk (Quillen et al. 1994). Also, we can take into account the gradient of the vertical thickness of the disk in the case of boxy or peanut-shaped structures (Laurikainen \& Salo 2002; Díaz-García et al. 2016) and the contribution of the dark matter halo (Buta et al. 2004; Díaz-García et al. 2016). However, the final bar strength has turned out to be rather insensitive to all these considerations (Laurikainen \& Salo 2002; Díaz-García et al. 2016). The most substantial uncertainties are induced from the disk thickness and the dark matter halo effect. Each can be about 10 to $15 \%$ in the value of bar strength (Laurikainen \& Salo 2002; Díaz-García et al. 2016).

\section{RATIO MAP}

We define the polar coordinates $(r, \phi)$ in the twodimensional plane $z=0$, the central plane of the galaxy. The mean radial force $\left\langle F_{\mathrm{R}}(r)\right\rangle$ and the transverse force $F_{\mathrm{T}}(r, \phi)$ can be defined from the two-dimensional potential $\Phi(r, \phi) \equiv \Psi(x, y, z=0)$, where

$$
\left\langle F_{\mathrm{R}}(r)\right\rangle \equiv \frac{d \Phi_{0}(r)}{d r}
$$

and

$$
F_{\mathrm{T}}(r, \phi) \equiv\left|\frac{1}{r} \frac{\partial \Phi(r, \phi)}{\partial \phi}\right| .
$$

$\Phi_{0}$ is the $m=0$ Fourier component of the gravitational potential (Combes \& Sanders 1981; Buta \& Block
2001). The maximum transverse force to the mean radial force is defined as

$$
Q_{\mathrm{T}}(r) \equiv \frac{F_{\mathrm{T}}^{\max }(r)}{\left\langle F_{\mathrm{R}}(r)\right\rangle},
$$

where the maximum tangential force $F_{\mathrm{T}}^{\max }(r)$ is the maximum of $F_{\mathrm{T}}(r, \phi)$ along $\phi$. BB01 employed the twodimensional ratio map of the transverse-to-radial force $Q_{\mathrm{T}}(i, j)$ in the Cartesian grid where

$$
Q_{\mathrm{T}}(i, j)=\frac{F_{T}(i, j)}{\left\langle F_{R}(i, j)\right\rangle} .
$$

We call these $2 \mathrm{D}$ values of $Q_{\mathrm{T}}(i, j)$ simply the ratio map for a given galaxy and the $Q_{\mathrm{T}}$ value at each point $(i, j)$ the force ratio.

In Figure 1, we display the calculated results for NGC 5750 and UGC 06903 . Figure 1(a) shows the deprojected image of the galaxy in the $i$-band and Figure 1(b) the ratio map $Q_{\mathrm{T}}(i, j)$. The ratio map shows four bright regions formed by the force ratio that reaches a maximum or minimum near the end of the bar (Buta \& Block 2001; Laurikainen \& Salo 2002). BB01 called it a butterfly pattern, and reported it as the characteristic signature for a bar. However, if a galaxy has spiral arms, the patterns on the ratio map become more complex, like UGC 06903 (bottom row in Figure 1(b)).

We denoted the position of the local maximum $Q_{\mathrm{T}}$ in each quadrant by blue crosses (Figure 1(a)). When we overlay the blue crosses on the deprojected image (Figure 1(a)), they are located near the four corners of the bar. BB01 defined the bar strength of a galaxy as the bar force ratio $Q_{\mathrm{b}}^{c} \equiv 4^{-1} \sum_{i=1}^{4} Q_{\mathrm{T}, i}$, where $Q_{\mathrm{T}, i}$ indicates the maximum $Q_{\mathrm{T}}$ in the quadrant $i$. We used the superscript ' $c$ ' to denote that it is obtained from the ratio map in the Cartesian coordinates in the manner of BB01.

\subsection{Ratio Map in the Polar Coordinates}

We have tried a different approach. We converted this ratio map $Q_{\mathrm{T}}(i, j)$ from the Cartesian coordinates to the polar coordinates, i.e., $Q_{\mathrm{T}}(r, \phi)$ as a function of $r$ and $\phi$. The ratio map can be described in the polar coordinates as

$$
Q_{\mathrm{T}}(r, \phi)=\frac{F_{\mathrm{T}}(r, \phi)}{\left\langle F_{\mathrm{R}}(r)\right\rangle},
$$

where the radial force and tangential force are given by equation 6 and equation 7 . It makes the complex patterns on the Cartesian grid dramatically simple and intuitive.

In Figure 1(c), we present the ratio map $Q_{\mathrm{T}}(r, \phi)$ for the same galaxy. The four wings of the butterfly pattern in the Cartesian grid are transformed to the four 

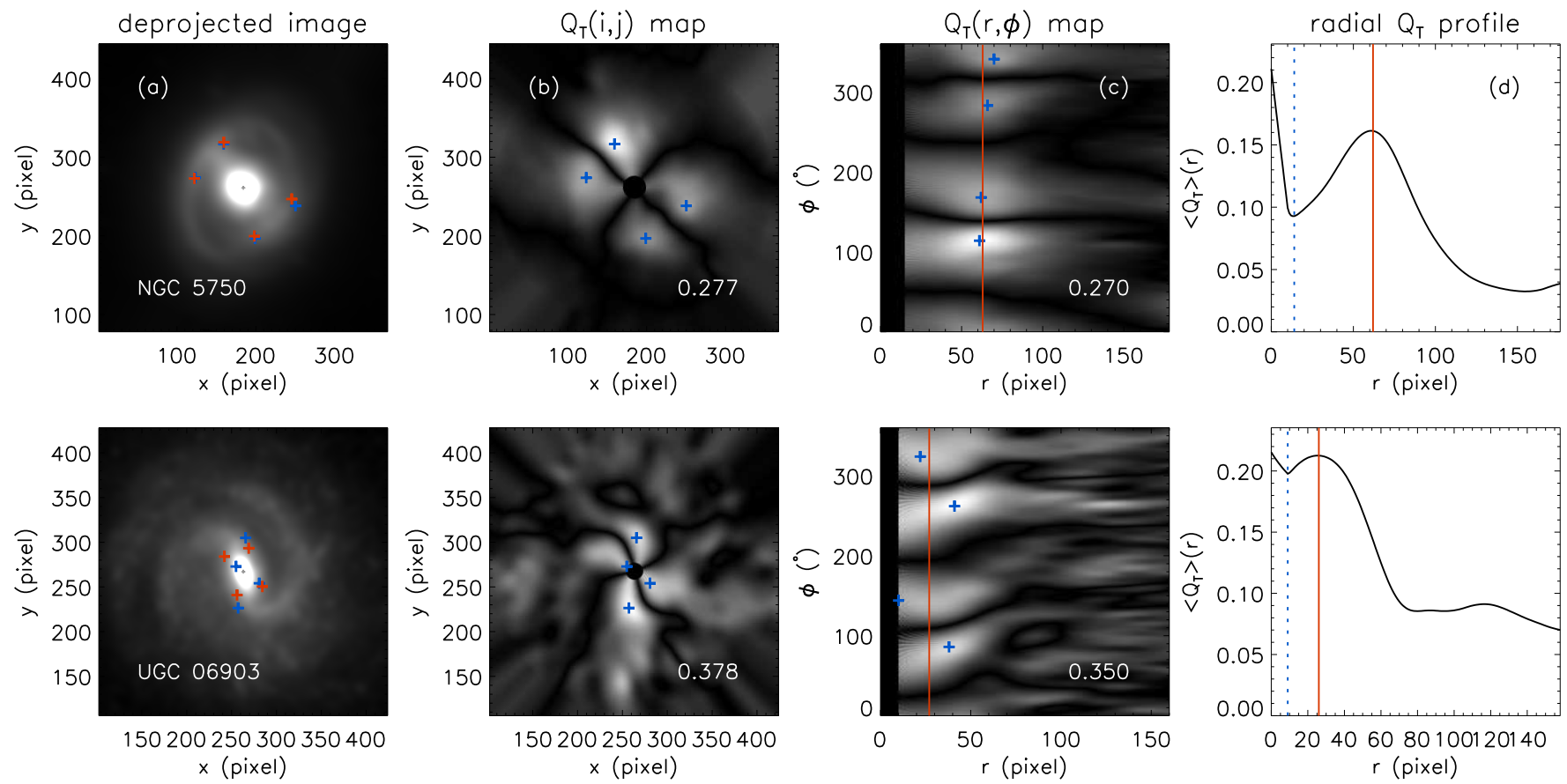

Figure 1. Examples of the transverse-to-radial force ratio map for NGC 5750 (upper row) and UGC 06903 (bottom row): (a) deprojected $i$-band image, (b) ratio map $Q_{\mathrm{T}}(i, j)$ in the Cartesian coordinates, (c) ratio map $Q_{\mathrm{T}}(r, \phi)$ in the polar coordinates, and (d) the radial $Q_{\mathrm{T}}$ profile $\left\langle Q_{\mathrm{T}}\right\rangle(r)$, averaged over the azimuthal angle at each radius. We find the bulge-dominated region (within blue vertical dotted line) and bar-dominated radius, $r_{\mathrm{Qb}}$ (red vertical solid line) on the radial $Q_{\mathrm{T}}$ profile. The bulgedominated region is excluded and the bar-dominated region is denoted by red vertical solid line on the $Q_{\mathrm{T}}(r, \phi)$ map. We overlay the maximum position of the force ratio in each quadrant from the Cartesian coordinates (blue cross) and those from the polar coordinates (red cross) on the deprojected $i$-band image and each ratio map. The bar strength $Q_{\mathrm{b}}^{c}$ from the Cartesian coordinates and $Q_{\mathrm{b}}$ from the polar coordinates are presented in the bottom right on each ratio map.

horizontal thick slabs, which are aligned with similar length $(r)$ and width $(\phi)$ in the $Q_{\mathrm{T}}(r, \phi)$ map. The four maxima in each quadrant (blue crosses in Figure 1(b)) appear within each thick slab in the $Q_{\mathrm{T}}(r, \phi)$ map (blue crosses in Figure 1(c)).

On the other hand, spiral arms are distinguished from the bar, appearing like ripples outside the bar (bottom row in Figure 1(c)). This is one of the most important merits of this representation because the BB01 method often has a trouble that maximum points in quadrants are influenced by a bulge or by spiral arms (Laurikainen \& Salo 2002; Buta et al. 2003, 2005; Garcia-Gómez et al. 2017). Buta et al. (2003) developed a way to separate the bar strength from the spiral strength by analyzing the additional Fourier amplitude. Meanwhile, we disentangle the bar strength from the strength caused by a bulge and spiral arms by investigating the ratio map as a function of $r$ and $\phi$.

Besides, we noticed that the four thick slabs for a bar are warped when they are connected to the ripples of the spiral arms. The degree of warping may provide the information about the pitch angle of the spiral arms. In fact, the ratio map shows all kinds of galactic struc- tures, a marble cake mixed with signatures from diverse components.

In Figure 1(d), we present the radial $Q_{\mathrm{T}}$ profile, which is the averaged $Q_{\mathrm{T}}$ over the azimuthal angle at each radius,

$$
\left\langle Q_{\mathrm{T}}\right\rangle(r)=\frac{1}{2 \pi} \int_{0}^{2 \pi} Q_{\mathrm{T}}(r, \phi) d \phi
$$

This graph has been investigated in previous studies by Laurikainen \& Salo (2002) and Laurikainen et al. (2004a,b). This profile shows decreasing mean strengths from the center, followed by an increase to the maximum. The range from the center to the first minimum is the bulge-dominated region, denoted by the blue vertical dotted line. As the bar dominates, $\left\langle Q_{\mathrm{T}}\right\rangle(r)$ starts to increase and has a maximum peak, indicated by the red vertical solid line. We call this $r_{\mathrm{Qb}}$, the radius where $\left\langle Q_{\mathrm{T}}\right\rangle(r)$ reaches a maximum. This radius has been studied and used as one type of measurements for the bar length (Laurikainen et al. 2002; Laurikainen \& Salo 2002; Laurikainen et al. 2004a,b; Díaz-García et al. 2016). 
Now, we investigate the marble cake map of $Q_{\mathrm{T}}(r, \phi)$ in $(r, \phi)$ coordinates (Figure 1(c)). First, we exclude the bulge region in the ratio map, shown as a black vertical stripe, in order to highlight the bar region. Then, we overlay $r_{\mathrm{Qb}}$ shown as the red solid line on the $Q_{\mathrm{T}}(r, \phi)$ map. It is located near the four maxima (blue crosses) used by BB01. Cutting this marble cake of $Q_{\mathrm{T}}(r, \phi)$ at $r_{\mathrm{Qb}}$ yields the azimuthal profile of $Q_{\mathrm{T}}$ at $r_{\mathrm{Qb}}$, i.e., the variation of $Q_{\mathrm{T}}\left(r_{\mathrm{Qb}}, \phi\right)$ along the azimuth $\phi$. We plot the azimuthal profile of UGC 06903 in Figure 2 and find four maximum peaks, each corresponding to the maximum force ratio at each quadrant. We again overlay these four peaks on the deprojected image with red crosses in Figure 1(a). They are located near the four maxima obtained from the BB01 procedures.

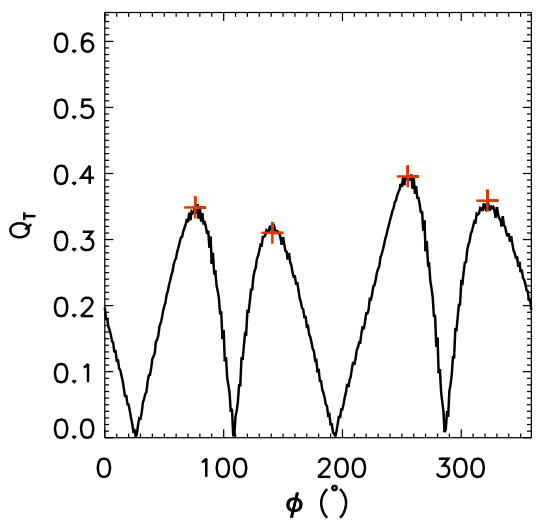

Figure 2. Azimuthal profile $Q_{\mathrm{T}}\left(r_{\mathrm{Qb}}, \phi\right)$ of UGC 06903 (Figure 1) at $r_{\mathrm{Qb}}$, where the maximum of the radial profile $\left\langle Q_{\mathrm{T}}\right\rangle(r)$ is located. The butterfly pattern appears as four peaks (red crosses) along the azimuth $\phi$.

We define, as the global bar strength measure, the bar force ratio of a galaxy in the polar coordinate representation as

$$
Q_{\mathrm{b}} \equiv \frac{1}{m} \sum_{i=1}^{m} Q_{\mathrm{T}, i}
$$

where $Q_{\mathrm{T}, i}$ is the maximum value at each peak on the azimuthal profile $Q_{\mathrm{T}}\left(r_{\mathrm{Qb}}, \phi\right)$ at $r_{\mathrm{Qb}}$, and $m$ is the number of the peaks. For typical bar structures, the value of $m$ is usually four, but in the presence of other structures, $m$ might be different from four. We use $Q_{\mathrm{b}}$ and $m$ values to classify barred galaxies in $\S 4.2$.

In Figure 3, we compare our bar force ratio value $Q_{\mathrm{b}}$ with $Q_{\mathrm{b}}^{c}$ measured by the BB01 method. They are quite correlated, but the values of $Q_{\mathrm{b}}^{c}$ are always greater compared to $Q_{\mathrm{b}}$. Theoretically, it is because the two bar force ratio measurements, $Q_{\mathrm{b}}^{c}$ and $Q_{\mathrm{b}}$, are different in that $Q_{\mathrm{b}}^{c}$ is the mean of global maxima of $Q_{\mathrm{T}}$ while $Q_{\mathrm{b}}$

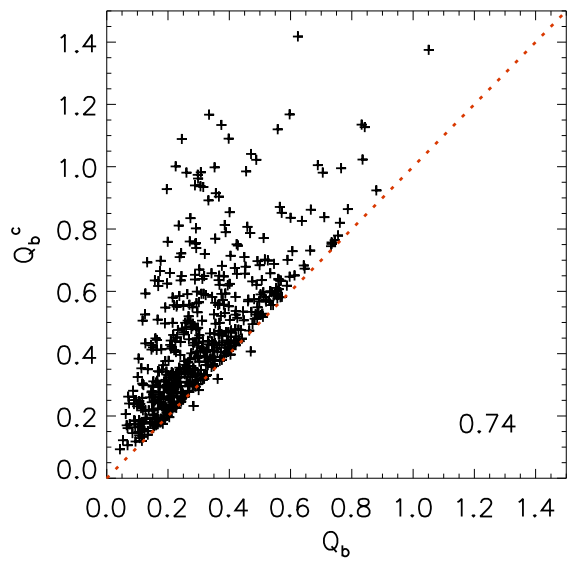

Figure 3. Comparison of bar force ratio measurements in the polar coordinates $\left(Q_{\mathrm{b}}\right)$ with that in the Cartesian coordinates $\left(Q_{\mathrm{b}}^{c}\right)$. The correlation coefficient is at the bottom right. The red dotted line denotes the track of a one-to-one correspondence.

is the mean of the maxima of $Q_{\mathrm{T}}$ at a given radius $r_{\mathrm{Qb}}$. However, we found that, in a large number of galaxies, the global maxima are contaminated by a bulge or spiral arms, as noted previously (Laurikainen \& Salo 2002; Buta et al. 2003, 2005; Garcia-Gómez et al. 2017). The local maximum derived at a certain radius helps the bar force ratio measurements not to be confused with other components, such as a bulge or spiral arms.

\subsection{Test on Mock Galaxies}

We constructed simple mock galaxies using GALFIT (Peng et al. 2010) to test the properties of the $Q_{\mathrm{T}}(r, \phi)$ map before applying to observed galaxies. Mock galaxies are generated to have a bulge and a disk with or without a bar. The bulge is built with the Sérsic index $n=2$ and the disk component has an exponential radial profile. We considered two types of bars: a flat bar with a constant radial profile and an exponential bar with exponentially decreasing radial profile (Elmegreen \& Elmegreen 1985; Kim et al. 2015). Flat bars are more related to strongly barred galaxies. They are longer and stronger and have higher contrasts compared to exponential bars (Elmegreen \& Elmegreen 1985, 1989; Baumgart \& Peterson 1986; Elmegreen et al. 1996; Regan \& Elmegreen 1997; Kim et al. 2015; Lee et al. 2019). We have built flat bars with the Sérsic index $n_{\text {bar }}=0.25$ and exponential bars with $n_{\text {bar }}=$ 0.85 , which are the mean values of $n_{\text {bar }}$ from flatbarred and exponential-barred galaxies, respectively (Kim et al. 2015). We have fixed the axis ratio of the bar to 0.3 . 

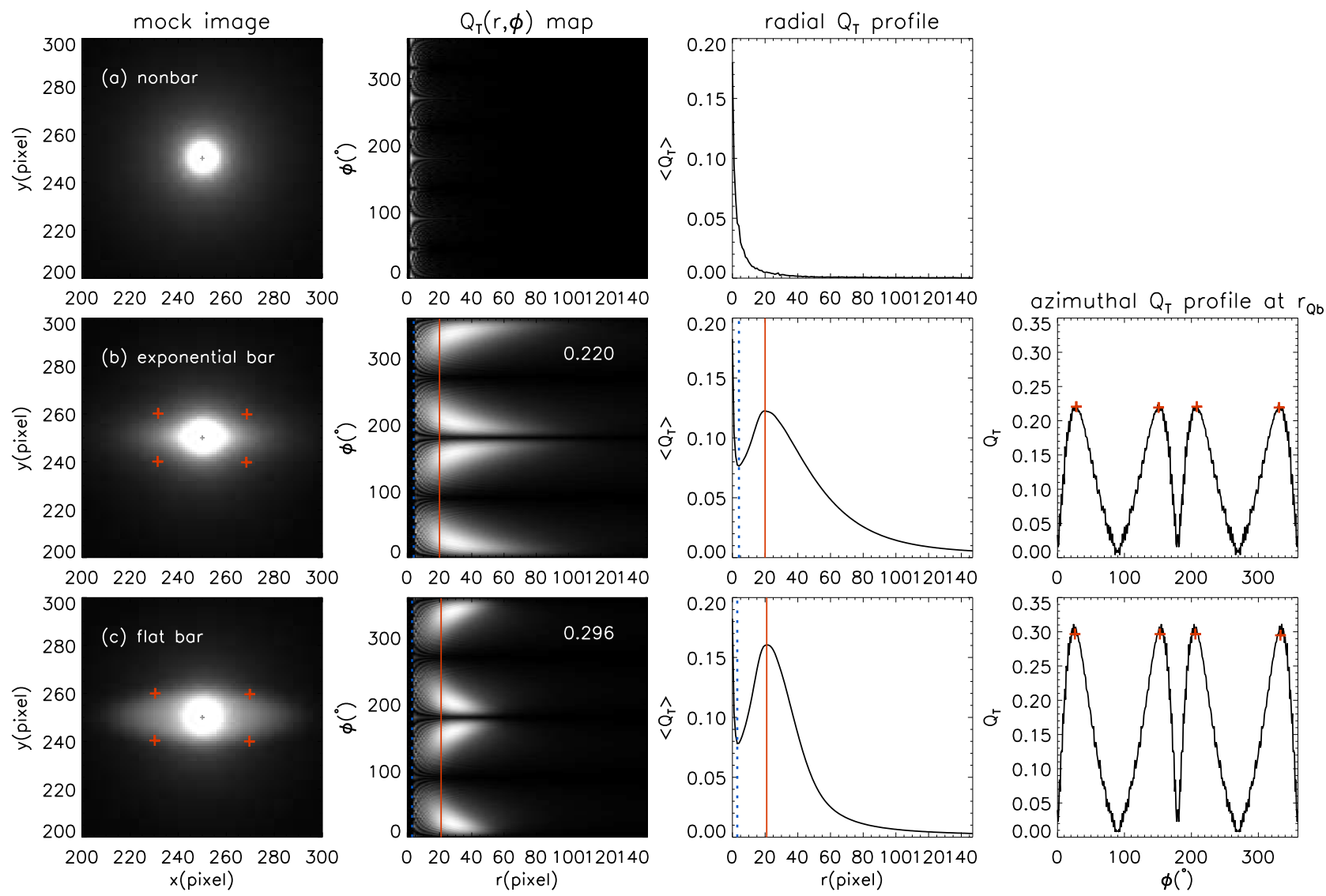

Figure 4. Test on mock galaxies: (a) a nonbarred galaxy, (b) an exponential-barred galaxy, and (c) a flat-barred galaxy. All images contain a bulge with the bulge-to-total light ratio of 0.2 and a disk. From left to right, the mock image, ratio map $Q_{\mathrm{T}}(r, \phi)$, radial profile $\left\langle Q_{\mathrm{T}}\right\rangle(r)$, and azimuthal profile $Q_{\mathrm{T}}\left(r_{\mathrm{Qb}}, \phi\right)$ at $r_{\mathrm{Qb}}$ are presented. Blue vertical dotted lines and red vertical solid lines present the minimum and maximum radius of $\left\langle Q_{\mathrm{T}}\right\rangle$, respectively. The inner region of the minimum radius of $\left\langle Q_{\mathrm{T}}\right\rangle$ is presented in black on the $Q_{\mathrm{T}}(r, \phi)$ map for barred galaxies to emphasize the bar pattern. Red crosses indicate peaks on the azimuthal profile at $r_{\mathrm{Qb}}$. We denote the bar force ratio $Q_{\mathrm{b}}$ on the top right in the second panel, the mean value of the peaks over the azimuthal angle at $r_{\mathrm{Qb}}$.

Figure 4 shows mock galaxies without a bar, with an exponential bar, and with a flat bar, from top to bottom. All have the same bulge-to-total ratio of 0.2 . We present in Figure 4 the mock image, $Q_{\mathrm{T}}(r, \phi)$ map, radial profile $\left\langle Q_{\mathrm{T}}\right\rangle(r)$, and azimuthal profile $Q_{\mathrm{T}}\left(r_{\mathrm{Qb}}, \phi\right)$ at $r_{\mathrm{Qb}}$ from left to right for each example. We confirm that the butterfly patterns are converted to four aligned thick slabs on the $Q_{\mathrm{T}}(r, \phi)$ map for both flat-barred and exponential-barred galaxies (second panels from left in Figures 4(b) and (c)). On the other hand, a mock galaxy without a bar shows a totally different pattern on the $Q_{\mathrm{T}}(r, \phi)$ map (second panel from left in Figure 4(a)). It shows a non-vanishing $Q_{\mathrm{T}}$ pattern only near the center, where digitizing errors produce artificial transverse forcing. We subtract this pattern caused by a bulge on other $Q_{\mathrm{T}}(r, \phi)$ maps throughout this paper, to highlight the patterns by bars.

The radial profile $\left\langle Q_{\mathrm{T}}\right\rangle(r)$ of a nonbarred galaxy is also quite different from those of both types of barred galaxies (third panels from left in Figure 4). The radial profile gradually decreases in the nonbarred galaxy (Figure 4(a)). However, when the mock galaxies have a bar, the radial profiles decrease from the center to reach a minimum, then followed by a maximum (Figures 4(b) and (c)). We regard the range from the center to the minimum as the bulge-dominated region and the region around the maximum as the bar-dominated region. Flat bars have a larger maximum value than exponential bars.

In the fourth panels from left, we display the azimuthal profile of $Q_{\mathrm{T}}\left(r_{\mathrm{Qb}}, \phi\right)$ at $r_{\mathrm{Qb}}$. Four wings of the 
butterfly pattern for a bar appear as four peaks on the azimuthal profiles (Figures 4(b) and (c)). We overlay the four peaks as red crosses on the mock images in the first panels and display the bar force ratio of a galaxy $Q_{\mathrm{b}}$ at the top right in the second panels. The flat-barred galaxy has a larger bar force ratio than the exponentialbarred galaxy when they have the same bulge and disk. For the nonbarred mock galaxy (Figure 4(a)), we cannot display the azimuthal profile at $r_{\mathrm{Qb}}$, because it does not have any maximum peak on the radial profile, except for the center.

\section{CLASSIFICATION}

\subsection{Two Types of the Radial Profile}

Now we analyze $Q_{\mathrm{T}}(r, \phi)$ maps for our 884 sample galaxies. The first step in automatically finding the bar signature is to determine $r_{\mathrm{Qb}}$ on the radial profile. In Figure 5, we display representative examples that have similar features shown in the analysis of mock barred galaxies (Figure 4). Although the shapes of radial profiles are not the same, all have a minimum point followed by a local maximum peak (Figure $5(\mathrm{c})$ ). We measure $r_{\mathrm{Qb}}$ at the local maximum peak in this case, denoted by the red vertical solid line.

On the other hand, we find another type that does not have a local maximum in the bar region (Figure 6(c)). Their radial profiles $\left\langle Q_{\mathrm{T}}\right\rangle(r)$ have a kind of plateau instead of a local maximum. When we cut the marble cake of $Q_{\mathrm{T}}(r, \phi)$ at the inflection radius where a plateau resides,

$$
\frac{d^{2}\left(\left\langle Q_{\mathrm{T}}\right\rangle(r)\right)}{d r^{2}}=0,
$$

we also find four peaks, the signature for a bar on the azimuthal profile, as shown in Figure 6(d). We present the four peaks with red crosses on the deprojected images and confirm that they are located around the four corners of the bar (Figure 6(a)). Hence, in such case, we define $r_{\mathrm{Qb}}$ as the radius of inflection. We indicate $r_{\mathrm{Qb}}$ by red vertical solid lines in Figures 6(b) and (c).

We investigate images for sample galaxies of these two types by visual inspections, and notice that more developed barred galaxies with grand-design spiral arms or rings usually have a maximum peak in their radial profiles. On the other hand, galaxies with a plateau in their radial profiles rarely show prominent structures, except for bars. We suspect that the different features in the radial profile might be related to the stages in bar evolution. We further investigate these two types in comparison with the analysis for the numerical simulation from Seo et al. (2019) and discuss it in §6.3. Here, we roughly classify galaxies into two types on the radial profiles $\left\langle Q_{\mathrm{T}}\right\rangle(r)$ : one that has a local maximum peak and the other that has a plateau with an inflection point. We call the former 'type M', standing for 'maximum peak', and the latter 'type P', signifying to the 'plateau'.

Naturally, there are other diverse cases in real galaxies. NGC 5584 and NGC 5705 have not only a plateau but also a local maximum in the radial profile, which are denoted by red solid and dotted lines, respectively, in Figures 6(b) and (c). Similarly, there are some galaxies that have two local maximum peaks. For these cases, we investigate all azimuthal profiles for a plateau or local maximum peaks. For example, in Figure 7, we compare the azimuthal profiles of NGC 5584 at both radii of a plateau and of a local maximum. The profile at the plateau has four peaks for a bar signature (Figure $7(\mathrm{~b})$ ), whereas that at the local maximum shows five peaks (Figure $7(\mathrm{c})$ ). We overlay each peak on the deprojected image by red crosses and skyblue triangles in Figure 7(a). We confirm that the five peaks at the local maximum are caused by spiral arms. We calculate the mean value $\left\langle Q_{\mathrm{T}, i}\right\rangle$ of peaks on the azimuthal profile at each radius (equation 12) and denote it at the top right of each panel in Figure 7. Even though the $\left\langle Q_{\mathrm{T}, i}\right\rangle$ value at the local maximum peak is larger than that at the plateau, we conclude that the large value is caused by spiral arms, not by the bar. Therefore, we preferentially adopt $r_{\mathrm{Qb}}$ where the azimuthal profile shows the bar signature, i.e., four peaks.

\subsection{Two Classes on the Azimuthal Profile: Bar versus Nonbar}

As discussed above, we can distinguish bars from spiral arms on the $Q_{\mathrm{T}}(r, \phi)$ map: bar structures appear as four thick horizontal slabs, whereas spiral arms as ripples. It becomes more apparent when we investigate the azimuthal profiles at their $r_{\mathrm{Qb}}$, as shown in Figure 7 . Bars have exactly four peaks while spiral arms usually have more than four peaks. It is the most essential criterion in distinguishing barred galaxies from nonbarred galaxies. We further constrain $Q_{\mathrm{b}}$ to be greater than a certain threshold $\left(Q_{\mathrm{b}} \geq 0.15\right)$ in order to exclude very weak ovals or elongated bulges. Some of the elongated bulges in the deprojected images are artifacts due to simple deprojection of spherical bulges.

We classify a galaxy as a barred galaxy if it has (1) four peaks in the azimuthal profile of $Q_{\mathrm{T}}$ at $r_{\mathrm{Qb}}$ and (2) the bar strength $Q_{\mathrm{b}}$ above 0.15 . Using these criteria, we classified 468 spirals as barred galaxies (53\%) and 416 spirals as nonbarred galaxies $(47 \%)$. This bar fraction is close to the bar fraction (60\%) including both SBs and SABs by the classical visual inspection (Nilson 1973; Sandage \& Tammann 1987; de Vaucouleurs et al. 1991; Buta et al. 2015; 

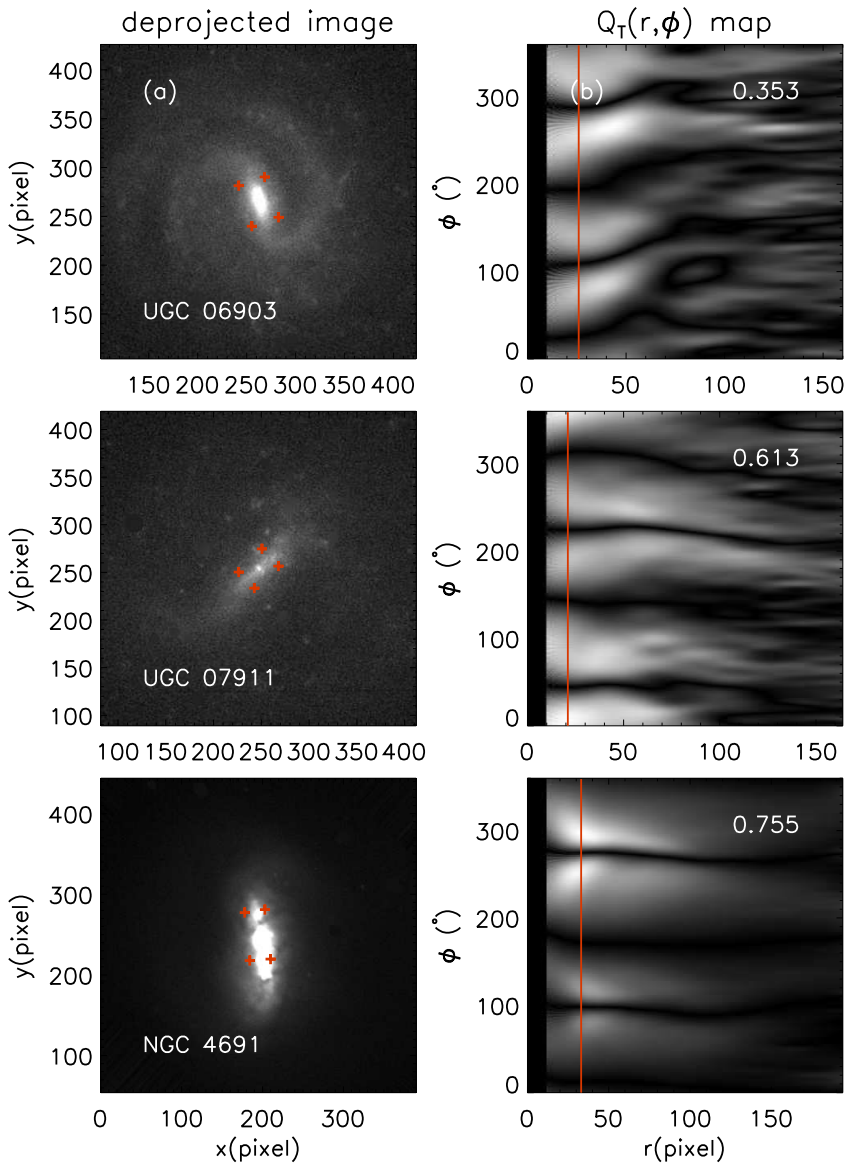
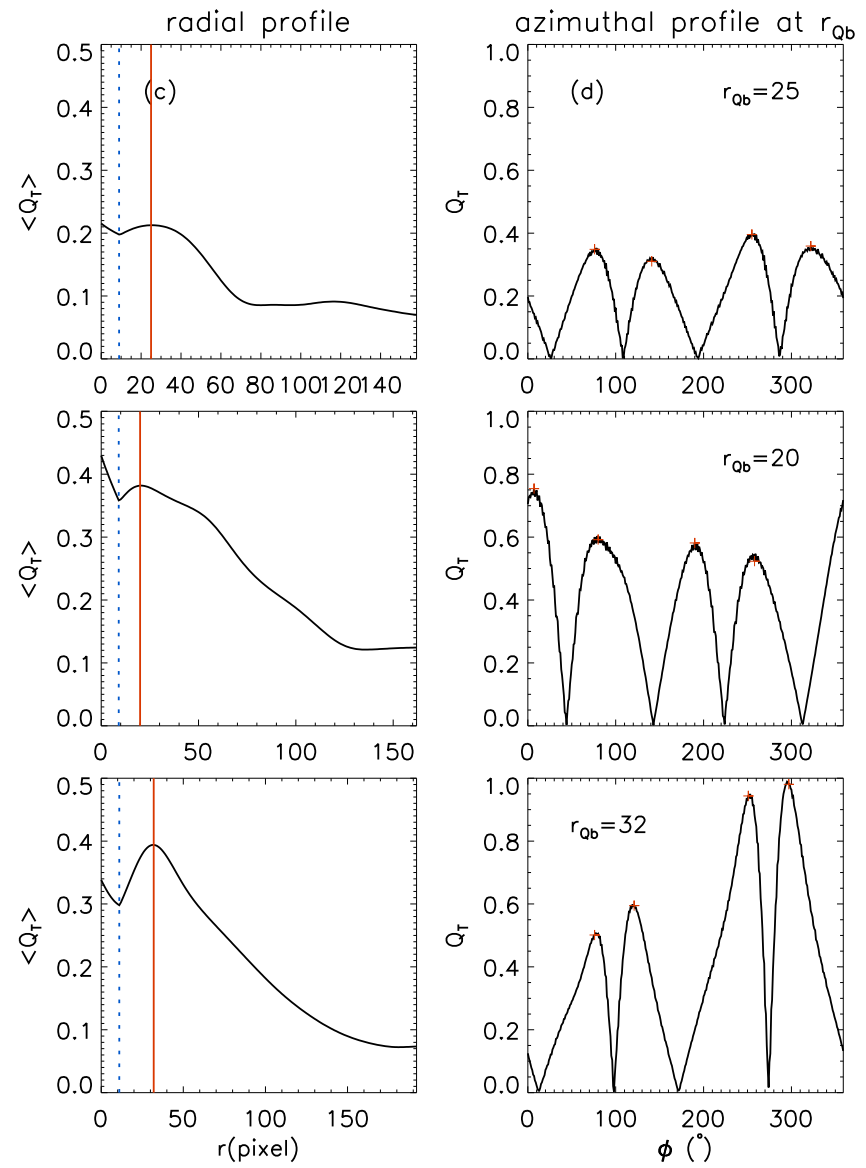

Figure 5. Example type $M$ galaxies, which have a local maximum peak on the radial profile. For each galaxy, we present (a) the deprojected image, (b) $Q_{\mathrm{T}}(r, \phi)$ map, and (c) the radial and (d) azimuthal profile of $Q_{\mathrm{T}}$. Blue vertical dotted lines in the panel (c) indicate the end of the bulge-dominated region. Red vertical solid lines present $r_{\mathrm{Qb}}$, corresponding to the bar-dominated region. Red crosses indicate peaks on the azimuthal profile at $r_{\mathrm{Qb}}$. The bar strength $Q_{\mathrm{b}}$ is denoted on the top right in the panel (b).

Ann et al. 2015), but is higher than that by previous automated classifications such as the ellipse fitting method $(\sim 45 \%)$ or Fourier analysis $(\sim 40 \%)$ (Laurikainen \& Salo 2002; Marinova \& Jogee 2007; Reese et al. 2007; Barazza et al. 2008; Aguerri et al. 2009; Marinova et al. 2010; Lee et al. 2019).

\subsection{Comparison with the Visual Classification}

We compare our new automated classification method against the visual inspection. For comparison, we used $63 \mathrm{SA}, 64 \mathrm{SAB}$, and $84 \mathrm{SB}$ galaxy 'concordance' sample in Paper I. It is the sample of galaxies classified as the same class by two independent visual inspections, RC3 (de Vaucouleurs et al. 1991) and Ann15. Figure 8 shows the consistency between our automated classification (ordinate) and the visual classification (abscissa). Our method shows good agreements with the visual classification by $79 \%$ and $87 \%$ for the SA and SB classes, respectively. However, our method classifies about half of visually classified SABs as SBs and the other half as
SAs, which is understandable because SABs by definition show weak bar features.

We display the example of the matched and unmatched galaxies in Figure 9. The galaxies in the upper row are barred galaxies (SBs) determined by our $Q_{\mathrm{T}}(r, \phi)$ map analysis and those in the bottom row nonbarred galaxies (SAs). The visual classification is displayed as $\mathrm{SA}, \mathrm{SAB}$, and $\mathrm{SB}$ in the abscissa from left to right. For each galaxy, we present the deprojected image, $Q_{\mathrm{T}}(r, \phi)$ map, and the radial and azimuthal profile at $r_{\mathrm{Qb}}$ from top to bottom.

We confirm that the azimuthal profiles of the upper row are definitely different from those of the bottom row, although they are unmatched against the visual classification. Whereas the azimuthal profiles on the upper row show exactly four peaks, those on the bottom row show more or less than four peaks. The shapes of peaks look different as well: the peaks of the upper row look regular and similar to each other, whereas those of the 

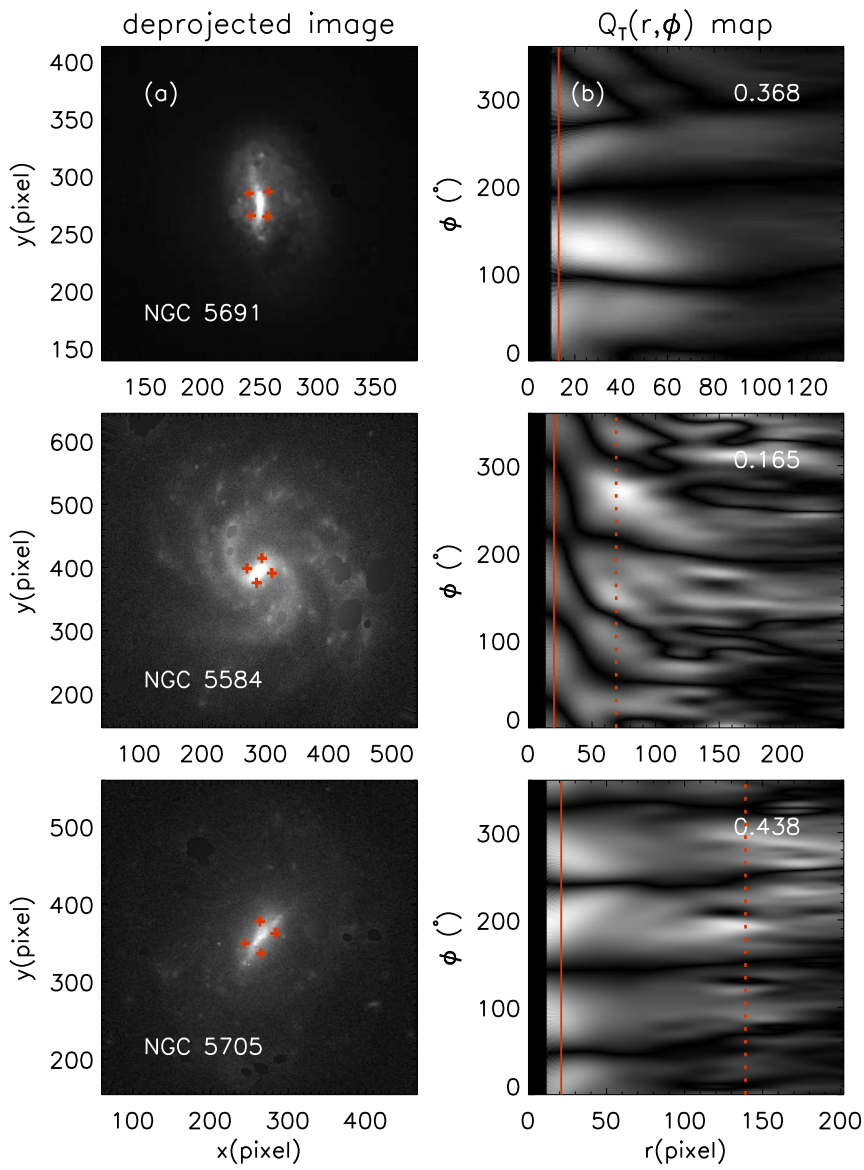
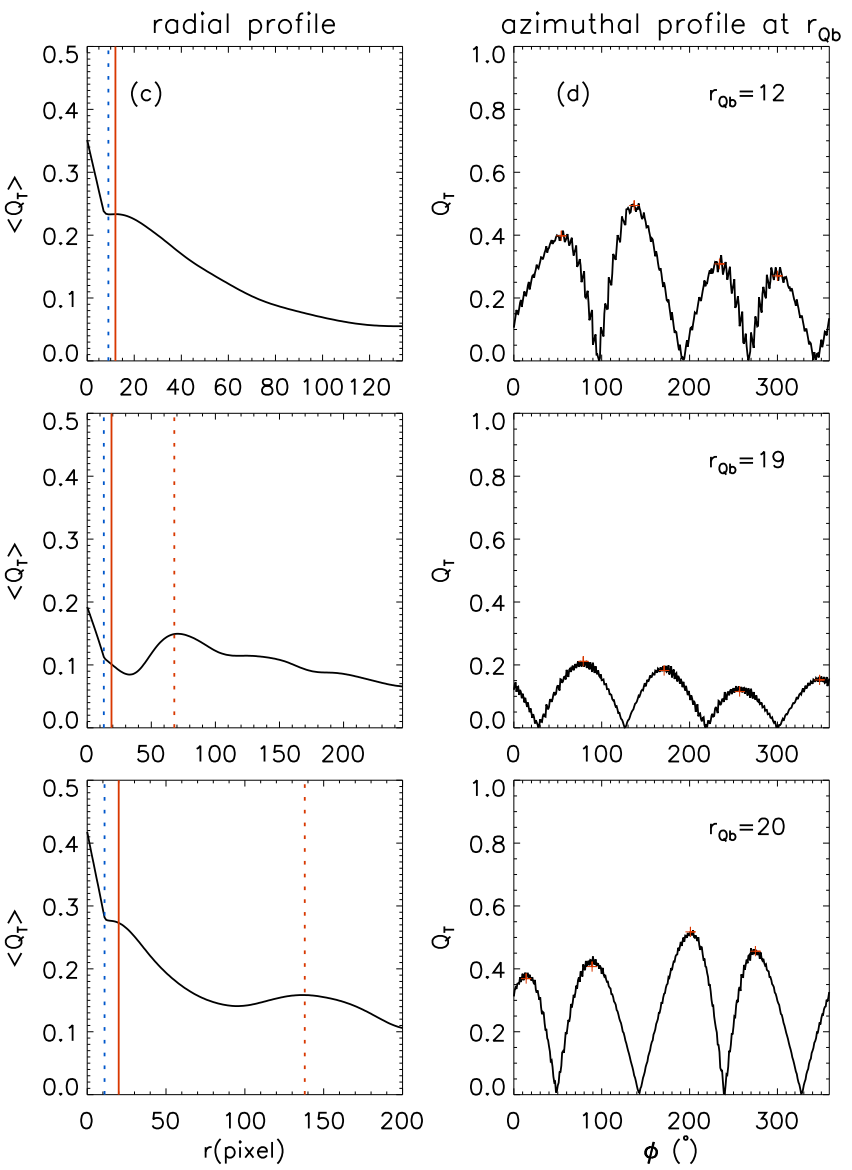

Figure 6. Example type $\mathrm{P}$ galaxies, which has a plateau in the bar region in the radial profile. For each galaxy, we present (a) the deprojected image, (b) $Q_{\mathrm{T}}(r, \phi)$ map, and (c) the radial and (d) azimuthal profile of $Q_{\mathrm{T}}$. As in Figure 5, blue dotted lines, red solid lines, and red crosses indicate the limit of the bulge-dominated region, $r_{\mathrm{Qb}}$, and four peaks of a bar at $r_{\mathrm{Qb}}$, respectively. In the cases that the radial profile has not only a plateau but also a local maximum peak, we mark the radius for a local maximum with the red vertical dotted line. The bar strength $Q_{\mathrm{b}}$ is displayed on the top right in the panel (b).

bottom row look very irregular. In fact, we found four additional barred galaxies that the previous visual inspections did not find, as shown in Figure 9(a), and a galaxy that has oval structures, as shown in Figure 9(b), among the unmatched galaxies (13 galaxies) against visually determined nonbarred galaxies. Accordingly, our misjudgement against nonbarred galaxies becomes $13 \%$ (eight galaxies) in practice, not $21 \%$.

The main factor to cause the confusion is the deprojection process. If the inclination is underestimated, the less-deprojected disk may be confused as a bar. Overestimated inclination, on the other hand, stretches the disk along the minor axis. Therefore, we need to develop a more careful deprojection process to deal correctly with the spherical component, i.e., bulge, in order to increase the accuracy of the classification.

We missed 11 visually determined SB galaxies (13\%). One of them is disturbed by the residuals after masking bright clumpy sources, and three have $Q_{\mathrm{b}}$ values lower than 0.15 because of a large bulge, which will be discussed in $\S 6.1$. Meanwhile, we found interesting features in the rest of them: they have asymmetric structures. In Figure 9(1), NGC 4416 has two nuclei within its bar structure. The peak deviates significantly from the centroid. We denote the peak by a gray cross on the deprojected image. If we use the centroid as the center instead of the peak for this galaxy, we find the normal signature for a bar on the $Q_{\mathrm{T}}(r, \phi)$ map and the azimuthal profile. We found six other asymmetric bars like UGC 07700 shown in Figure 9(k). They usually do not have any bulge but have small bright sources in the elongated bar-like structures. The nucleus is displaced from the center of the bar. These are galaxies with unusual dynamics.

Lastly, we investigate how the inclination of a galaxy influences the accuracy of our automated classification. We excluded galaxies inclined more than $60^{\circ}$ and constructed face-on galaxy images through the deprojec- 
(a) deprojected image

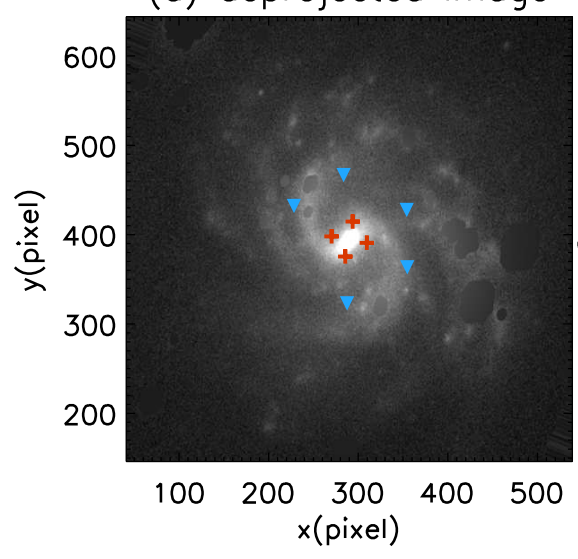

(b) at a plateau

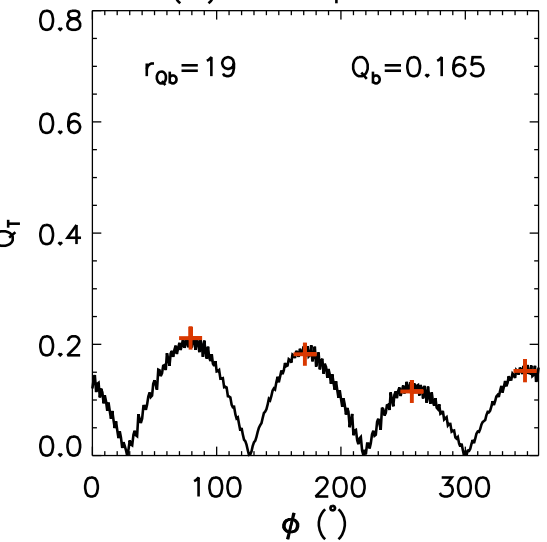

(c) at a local maximum

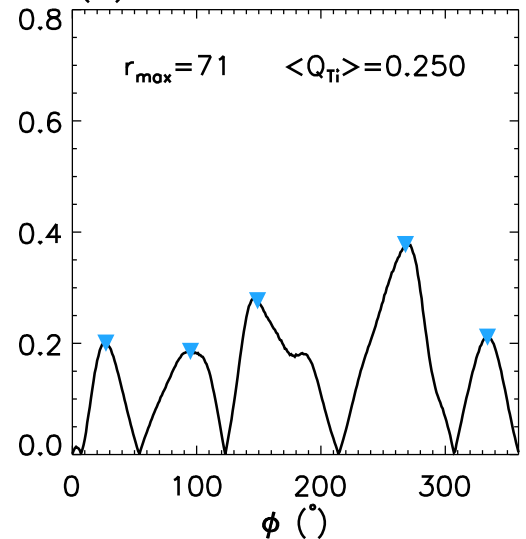

Figure 7. An example galaxy NGC 5584 that has both a plateau and a maximum peak on the radial profile. We present the deprojected image in (a) and azimuthal profiles at a plateau in (b) and a local maximum peak in (c). We display the radius of the plateau and the local maximum at the top left and the mean strength value of peaks at the top right in each panel. Then each peak on the azimuthal profiles is overlaid on the deprojected image by red crosses and skyblue triangles. The four peaks at the plateau are located near the bar and five peaks at the maximum radius in the middle of spiral arms.

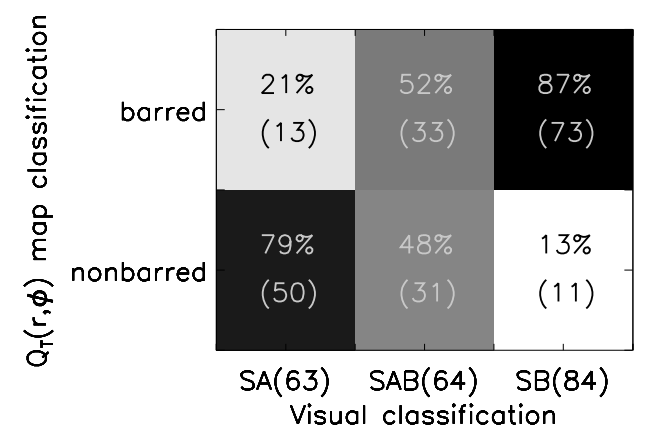

Figure 8. Comparison between our automated classification utilizing $Q_{\mathrm{T}}(r, \phi)$ map and the visual classification from RC3 (de Vaucouleurs et al. 1991) and Ann15 catalogs. The numbers mean matched percentages of the automated classification (ordinate) for the visual classification (abscissa). The larger number and darker shade indicate better agreement.

tion. We found that inadequate deprojection is the main factor causing mistakes in our automated classification as mentioned before.

We then investigate the agreement and disagreement rate of our automated classification against the visual classification by restricting the sample galaxies to inclinations less than $60^{\circ}, 45^{\circ}$, and $30^{\circ}$. In Table 1 , we organize the results both for the concordance sample and the whole sample of galaxies. We find that the effect of the inclination on the classification accuracy appears in SAs, but not in SBs. For the whole sample, the agreement rates are improved by $5 \%$ and $4 \%$ as the inclination restriction is lowered from $60^{\circ}$ to $45^{\circ}$ and $30^{\circ}$. This is because the more a galaxy is inclined, the less accurately the inclination is measured. A spherical bulge makes the inclination underestimated in high inclination galaxies. Consequently, the less-deprojected disk is easily confused as a bar, as discussed before. For the concordance sample, the agreement rate for $45^{\circ}$ restriction is reduced compared to that for $60^{\circ}$ restriction, but it is not statistically meaningful because the corresponding sample is too small.

Strongly barred galaxies seem to be less affected by the galaxy inclination. In the whole sample, the agreement rate increases by $3 \%$ as the inclination restriction is lowered from $60^{\circ}$ to $45^{\circ}$, but barred galaxies are mainly misjudged for other reasons such as the residuals after masking, asymmetric bars, or lower $Q_{\mathrm{b}}$ than 0.15 as discussed before, not by the inclination or deprojection. We do not find elongated bulges by the deprojection in the misjudged SBs. They are already excluded from barred galaxies because they have $Q_{\mathrm{b}}$ lower than 0.15 . We do not find any variation in the agreement rate between $45^{\circ}$ and $30^{\circ}$ restriction.

\section{4. $S B$ versus $S A B$}

We can speculate that weak bars (SABs) will be distinguished from strong bars (SBs) in terms of the bar strength. However, it is not straightforward because the bar strengths of strong bars and weak bars are distributed over a wide range (Buta \& Block 2001; Block et al. 2001; Laurikainen \& Salo 2002; Buta et al. 2004; Cuomo et al. 2019). 


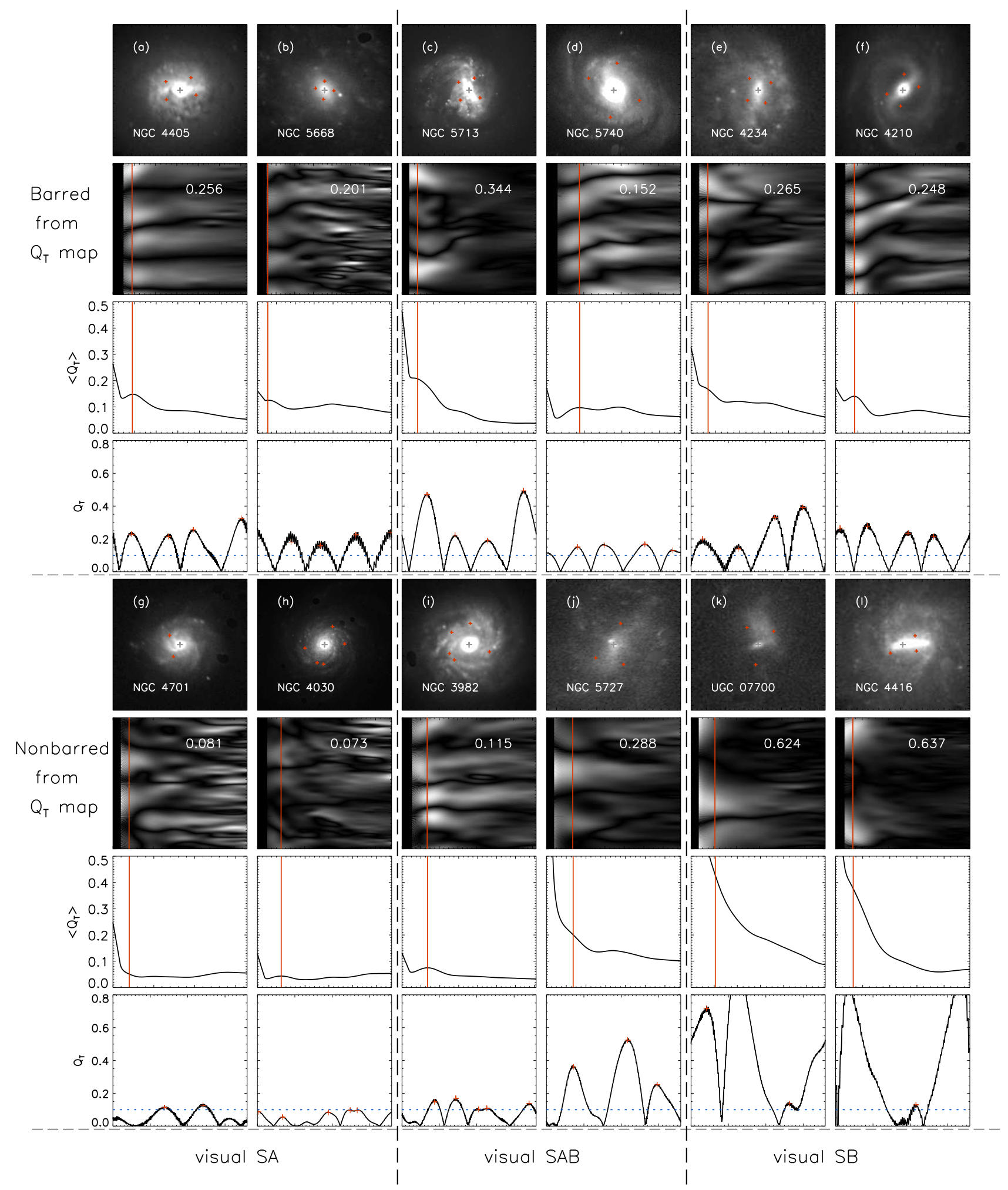

Figure 9. Representative example galaxies for comparison between automated classification by $Q_{\mathrm{T}}(r, \phi)$ map analysis and the visual classification from RC3 (de Vaucouleurs et al. 1991) and Ann15 catalogs. The upper row shows automatically classified SB galaxies and the bottom row SA galaxies. The visual classification is presented as SA, SAB, and SB in the abscissa from left to right. Each galaxy is shown with the deprojected image, $Q_{\mathrm{T}}(r, \phi)$ map, and the radial and azimuthal profile from top to bottom. 
Table 1. The Effect of the Inclination of Galaxy on the Agreement and Disagreement between the Visual Classification and the $Q_{\mathrm{T}}$ Map Classification

\begin{tabular}{|c|c|c|c|c|c|c|c|c|}
\hline & \multicolumn{2}{|c|}{ Inclination restriction } & \multicolumn{2}{|c|}{$60^{\circ}$} & \multicolumn{2}{|c|}{$45^{\circ}$} & \multicolumn{2}{|c|}{$30^{\circ}$} \\
\hline & \multicolumn{2}{|c|}{ Classification method } & \multicolumn{2}{|c|}{ Sample } & \multicolumn{2}{|c|}{ Sample } & \multicolumn{2}{|c|}{ Sample } \\
\hline & Visual inspection & $Q_{\mathrm{T}}$ map & $\operatorname{con}^{\mathrm{a}}(211)$ & all $^{\mathrm{b}}(884)$ & con (143) & all (560) & $\operatorname{con}(75)$ & all (271) \\
\hline \multirow{3}{*}{ Agreement } & $\mathrm{SA}$ & $\mathrm{SA}$ & $79 \%(50)$ & $67 \%(221)$ & $76 \%(32)$ & $72 \%(148)$ & $83 \%(20)$ & $76 \%(77)$ \\
\hline & $\mathrm{SAB}$ & SB & $52 \%(33)$ & $49 \%(143)$ & $56 \%(23)$ & $51 \%(94)$ & $53 \%(8)$ & $56 \%(45)$ \\
\hline & $\mathrm{SB}$ & $\mathrm{SB}$ & $87 \%(73)$ & $83 \%(218)$ & $88 \%(53)$ & $86 \%(148)$ & $89 \%(32)$ & $86 \%(77)$ \\
\hline \multirow{3}{*}{ Disagreement } & $\mathrm{SA}$ & $\mathrm{SB}$ & $21 \%(13)$ & $33 \%(107)$ & $24 \%(10)$ & $28 \%(57)$ & $17 \%(4)$ & $24 \%(24)$ \\
\hline & $\mathrm{SAB}$ & SA & $48 \%(31)$ & $51 \%(151)$ & $44 \%(18)$ & $49 \%(89)$ & $47 \%(7)$ & $44 \%(35)$ \\
\hline & $\mathrm{SB}$ & SA & $13 \%(11)$ & $17 \%(44)$ & $12 \%(7)$ & $14 \%(24)$ & $11 \%(4)$ & $14 \%(13)$ \\
\hline
\end{tabular}

The numbers in the parentheses indicate the number of galaxies.

a It means the concordance sample that are classified as the same

class by two independent visual inspections, RC3 and Ann15.

b It means the whole sample. 


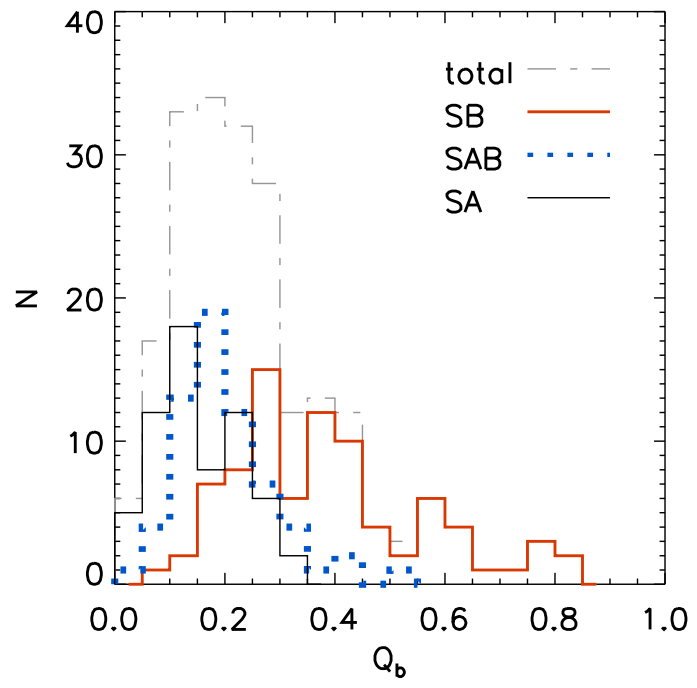

Figure 10. Distribution of the bar force ratio $Q_{\mathrm{b}}$ for visually classified SA, SAB, and SB galaxies. The gray dot-dashed line indicates total galaxies. We denote $\mathrm{SA}, \mathrm{SAB}$, and SB galaxies by thin black solid line, thick blue dotted line, and thick red solid line, respectively.

Figure 10 displays the distribution of the bar force ratio $Q_{\mathrm{b}}$ for visually determined $\mathrm{SA}, \mathrm{SAB}$, and $\mathrm{SB}$ glaxies. The force ratios for SA galaxies are defined by the mean values of the $Q_{\mathrm{T}}$ peaks along the azimuthal profile at $r_{\mathrm{Qb}}$ as well. The only difference from the barred galaxies is the number of peaks.

SB galaxies are known to have larger $Q_{\mathrm{b}}$ than SAB galaxies (Buta \& Block 2001; Buta et al. 2004, 2005, 2007): its distribution is statistically distinguishable from that of SABs. However, their values of the bar force ratio cover a wide range from $Q_{\mathrm{b}}=0.1$ to 0.8 as shown in Figure 10, which is consistent with earlier studies (Buta \& Block 2001; Block et al. 2001; Laurikainen \& Salo 2002; Buta et al. 2004). On the other hand, the bar force ratios of SAB galaxies are distributed in a narrower range than those of SB galaxies, and are similar to the distribution of force ratios for SAs rather than those for SBs (Figure 10), as shown in previous studies (Abraham \& Merrifield 2000; Laurikainen et al. 2004a; Garcia-Gómez et al. 2017). Nevertheless, it is hard to separate individual SAB galaxies from $\mathrm{SB}$ galaxies based directly on $Q_{\mathrm{b}}$ because they overlap in the distribution of bar force ratio. It could result from the fact that weak ovals may have a significant amount of mass.

Abraham \& Merrifield (2000) showed the separation between SB and SAB galaxies on the diagram of central concentration versus bar strength for a small sample of
56 galaxies. They defined the bar strength as

$$
S_{\mathrm{bar}}=\frac{2}{\pi}\left[\arctan (b / a)_{\mathrm{bar}}^{-1 / 2}-\arctan (b / a)_{\mathrm{bar}}^{+1 / 2}\right]
$$

where $\mathrm{a}$ and $\mathrm{b}$ are the major and minor axis of the bar, respectively. In Figure 11, we plot visually classified SB (red solid circle), SAB (blue square), and SA (black triangle) galaxies in the diagram of the light concentration $\left(C \equiv R_{90} / R_{50}\right)$ versus the bar force ratio $\left(Q_{\mathrm{b}}\right)$. We find similar distributions with Abraham \& Merrifield (2000) who showed a broad anti-correlation between the light concentration and the bar strength, and a skewed distribution of SAB toward lower $Q_{\mathrm{b}}$ compared to SB galaxies. However, we do not confirm the separation between SB and SAB galaxies. Even when we have used the bar strength (Equation 14) defined by Abraham \& Merrifield (2000), the result was not different.

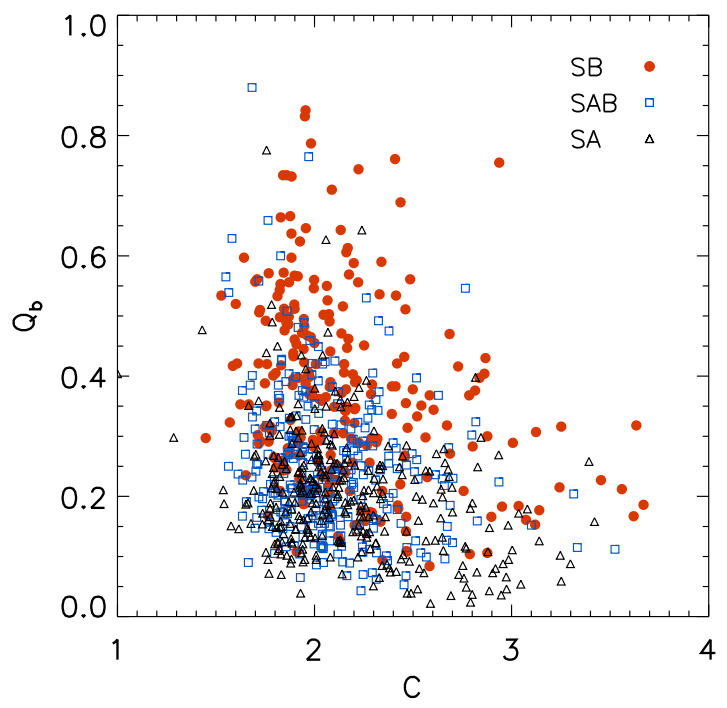

Figure 11. Distribution of visually classified SA (black triangle), SAB(blue square), and SB (red solid circle) galaxies in the diagram of the light concentration $\left(C \equiv R_{90} / R_{50}\right)$ versus the bar force ratio $Q_{\mathrm{b}}$.

Nevertheless, we attempted to classify barred galaxies into $\mathrm{SB}$ and $\mathrm{SAB}$ galaxies based on the value of $Q_{\mathrm{b}}$ alone. We set the criterion $0.15 \leq Q_{\mathrm{b}}<0.25$ for $\mathrm{SAB}$, which is the range that $\mathrm{SAB}$ galaxies are dominant as shown in Figure 10. We categorized galaxies with $Q_{\mathrm{b}} \geq 0.25$ as SBs. We classified the concordance sample galaxies used in Figure 8 according to this criterion. We present the consistency against the visual classification in Figure 12 as in Figure 8. This criterion classifies $36 \%$ of visually selected SAB galaxies as SABs. Although it is somewhat lower compared to SA or SB 
classes, it is the highest agreement for SABs when compared with other automated classification methods such as the ellipse fitting method shown in Paper I.

When we investigate the unmatched SABs, $16 \%$ are classified as SB because of $Q_{\mathrm{b}}$ larger than 0.25 , whereas $9 \%$ are classified as SAs due to $Q_{\mathrm{b}}$ smaller than 0.15. However, $39 \%$ of SABs do not satisfy the criterion of four peaks in the azimuthal profile of $r_{\mathrm{Qb}}$. It shows that many of SAB galaxies have characteristics similar to those of SA galaxies in terms of not only the bar strength but also of the pattern on the ratio map. This supports the previous speculations that $\mathrm{SAB}$ galaxies have a similar origin of the force ratio as SA galaxies (Laurikainen et al. 2004b) or that SAB galaxies would be the extension of SA galaxies (Abraham \& Merrifield 2000). It may also be that SAB galaxies are more heterogeneous than SB galaxies; visual SAB classification can refer to ovals as well as weak bars. In some galaxies, both an oval and a regular bar co-exist.

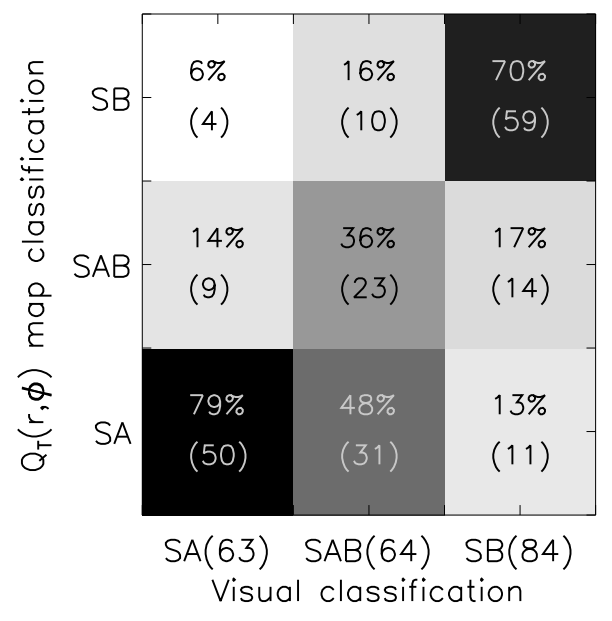

Figure 12. Comparison between our classification using bar force ratio $Q_{\mathrm{b}}$ and the visual classification from RC3 (de Vaucouleurs et al. 1991) and Ann15 catalogs. The numbers show matched percentages of the automated classification (ordinate) for the visual classification (abscissa).

\section{BAR PROPERTIES}

\subsection{Bar Strength and Length}

The $Q_{\mathrm{T}}(r, \phi)$ map analysis not only determines whether a bar is present or not but also provides important properties such as the bar strength $Q_{\mathrm{b}}$ and the bar length $r_{\mathrm{Qb}}$. The photometric bar strength $Q_{\mathrm{b}}$ is tightly correlated to the kinematic bar strength $Q_{\text {kin }}$ measured from the stellar velocity field (Seidel et al. 2015). Moreover, it is well correlated to other bar strength mea- surements such as the bar ellipticity $\epsilon_{\text {bar }}$ (Block et al. 2001; Laurikainen et al. 2002; Laurikainen \& Salo 2002; Díaz-García et al. 2016) and the normalized Fourier amplitude of $m=2$ over $m=0$ components, $A_{2}$ (Laurikainen et al. 2002; Díaz-García et al. 2016; Cuomo et al. 2019). The radius $r_{\mathrm{Qb}}$ has also been reported to be correlated to the bar length $r_{\text {vis }}$ estimated by visual inspection (Laurikainen et al. 2002; Díaz-García et al. 2016) and $r_{\mathrm{A} 2}$ by the Fourier analysis (Laurikainen et al. 2004a).

In Figure 13, we compare the various bar strength and length measurements by the $Q_{\mathrm{T}}(r, \phi)$ map analysis, ellipse fitting method, and Fourier analysis. In the ellipse fitting method, a bar is defined by the criteria on four parameters: the transition between a bar and a disk with $\Delta \epsilon_{\text {tra }} \geq 0.1$ and $\Delta \mathrm{PA}_{\text {tra }} \geq 5^{\circ}$, the ellipticity threshold of $\epsilon_{\mathrm{bar}} \geq 0.25$, and constant position angle $\Delta \mathrm{PA}_{\text {bar }}$ within $\pm 10^{\circ}$ (Jogee et al. 2004; Lee et al. 2019). For the Fourier analysis, the bar region should have the normalized Fourier intensity amplitudes larger than $A_{2}=0.3$ along with the $m=2$ and $m=4$ phases maintained constant (Ohta et al. 1990; Laurikainen et al. 2002; Lee et al. 2019). Although the definition of the normalized Fourier amplitude $A_{2}$ is somewhat different depending on the studies (Athanassoula et al. 2013; Seo et al. 2019), we use $A_{2}$ defined as $I_{2} / I_{0}$ throughout this paper, which is described by

$$
A_{2} \equiv \frac{I_{2}(r)}{I_{0}(r)}=\frac{\left[a_{2}(r)^{2}+b_{2}(r)^{2}\right]^{1 / 2}}{a_{0}(r) / 2}
$$

where $a_{0}, a_{2}$, and $b_{2}$ are the Fourier coefficients. (Ohta et al. 1990; Lee et al. 2019). The bar strength is defined by the maximum $\epsilon$ or $A_{2}$ in the bar region (Martin 1995; Laurikainen et al. 2002; Laurikainen \& Salo 2002), and the bar length $r_{\epsilon}$ or $r_{A 2}$ by the radius where the maximum of $\epsilon$ or $A_{2}$ occurs, which can be measured reliably, but is not the full length of the bar (Wozniak et al. 1995; Laurikainen et al. 2002; Laurikainen \& Salo 2002; Díaz-García et al. 2016). We used the $i$-band deprojected images for all methods in order to compare them in the same manner. For the ellipse fitting method, we used the criteria of $\epsilon_{\text {bar }} \geq$ 0.2 and $\Delta \mathrm{PA}_{\text {tra }} \geq 2^{\circ}$, which are the best criteria for the $i$-band deprojected images that yields the highest agreement with the visual classification (Paper I).

Figure 13 shows comparatively good correlations between different measurements of bar strength (upper row) and length (bottom row), as reported in the literature (Block et al. 2001; Laurikainen et al. 2002; Laurikainen \& Salo 2002; Díaz-García et al. 2016), although the scatters are not small. We find that the 

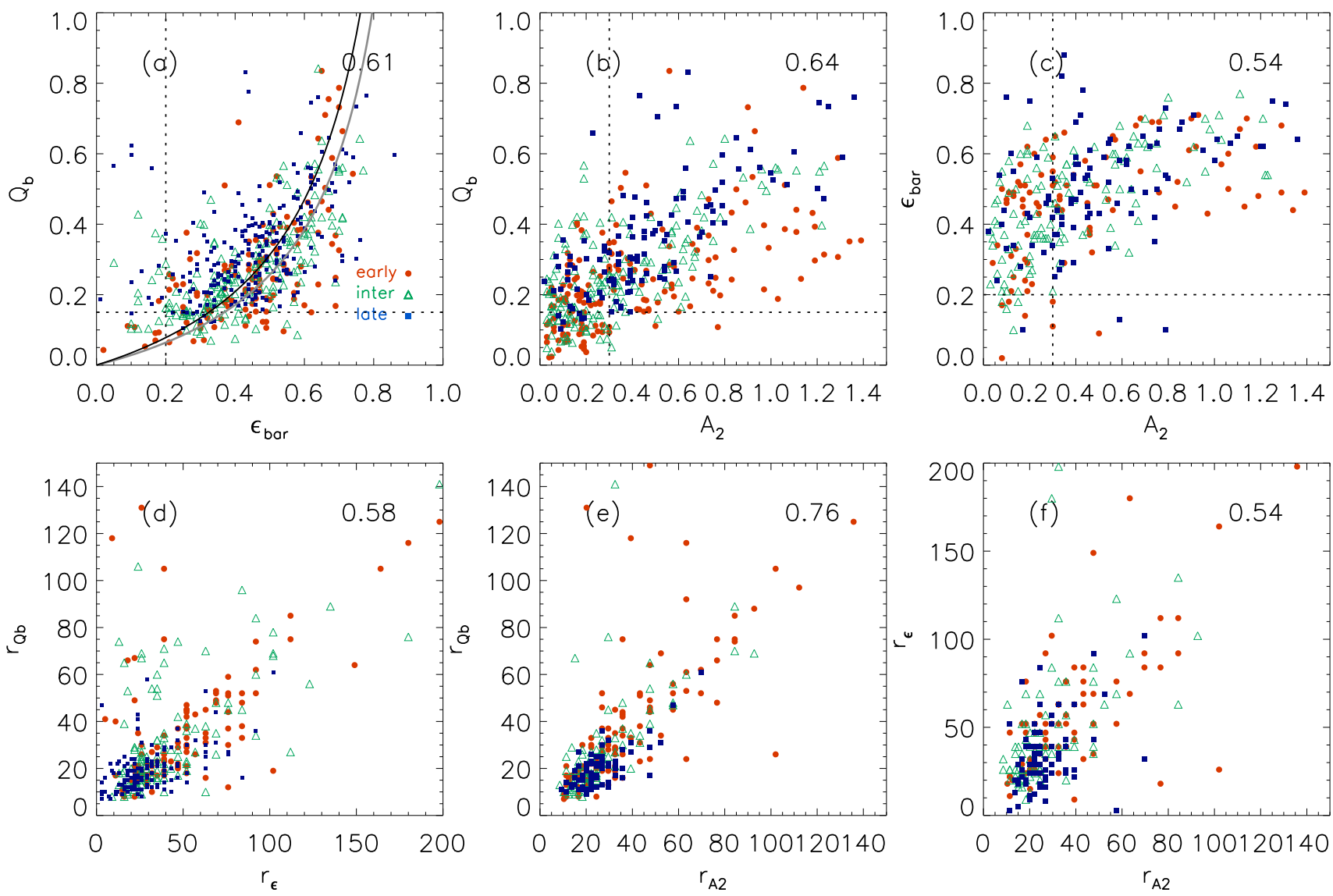

Figure 13. Comparison of bar strength (upper row) and length (bottom row) measurements between the $Q_{\mathrm{T}}(r, \phi)$ map analysis, ellipse fitting method, and Fourier analysis. In the upper row, we overplot the threshold $Q_{b}=0.15, \epsilon_{\mathrm{bar}}=0.2$, and $A_{2}=0.3$ for the bar detection of the three classification methods by the dotted lines. And the galaxies under the threshold are excluded in the bottom row. Galaxies are separated into early-type (red solid circle), intermediate-type (green open triangle), and late-type spirals (blue solid square). The correlation coefficients are presented at the top right of each panel. In the panel (a), we overplot the analytic relation between $\epsilon_{\mathrm{bar}}$ and $Q_{\mathrm{b}}$ (Kim et al. 2012), which depends on the bar mass fraction $f_{\mathrm{b}}$. The black and gray lines indicate the relations when $f_{\mathrm{b}}=0.5$ and 0.4 for a homogeneous bar $(n=0)$, respectively.

measurements from the $Q_{\mathrm{T}}(r, \phi)$ map and the Fourier analysis have the strongest correlations in both the bar strength (Figure 13(b)) and length (Figure 13(e)). The scatter in the bar strength is larger compared to that in the bar length. We separate galaxies into early-type (S0/a-Sb), intermediate-type (Sbc-Scd), and late-type $(\mathrm{Sd}-\mathrm{Sm})$ spirals by red solid circles, green open triangles, and blue solid squares, respectively, in Figure 13. We find a trend that for a given $Q_{\mathrm{b}}$ value early-type spirals are skewed to larger $A_{2}$ values, whereas late-type spirals to smaller $A_{2}$ values. These results have been shown in previous studies and explained by the presence of a large bulge in earlytype spirals and a dominant dark matter halo in latetype spirals (Laurikainen \& Salo 2002; Buta et al. 2004; Laurikainen et al. 2004a; Díaz-García et al. 2016). We will discuss it further in $\S 5.2, \S 6.1$, and $\S 6.2$.
On the other hand, the measurements by the ellipse fitting method and Fourier analysis are least-correlated in both the bar strength (Figure 13(c)) and the bar length (Figure 13(f)). The number of plotted samples is reduced because we only plot galaxies that satisfy the bar criteria in both methods.

The bar force ratio $Q_{\mathrm{b}}$ also has a good correlation with the bar ellipticity $\epsilon_{\text {bar }}$ (Figure $13(\mathrm{a})$ ), which reflects the fact that the underlying potential basically determines the orbits of stars (Díaz-García et al. 2016). It supports the proposition that the maximum ellipticity of a bar $\epsilon_{\text {bar }}$ can be a good approximation of the bar force ratio $Q_{\mathrm{b}}$ (Athanassoula 1992; Laurikainen et al. 2002; Laurikainen \& Salo 2002).

However, the relation of $Q_{\mathrm{b}}$ and $\epsilon_{\mathrm{bar}}$ looks different from those between other parameters that are roughly linear (Figures 13(b) and (c)). It is because the bar force ratio $Q_{\mathrm{b}}$ is related to the bar mass fraction and 
the orbit of stars (Kim et al. 2012). Kim et al. (2012) analytically derived a simple relation between $Q_{\mathrm{b}}$, the bar mass fraction $f_{\mathrm{b}}$, and the axis ratio $a / b$, where $a$ and $b$ are the semi major axis and minor axis, for a prolate Ferrers bar assuming a flat rotation curve. The force ratio $Q_{\mathrm{b}}$ turned out to be a nonlinear, yet simple, function of the bar mass fraction $f_{\mathrm{b}}$ for a given degree of central density concentration $c$ :

$$
\begin{aligned}
Q_{\mathrm{b}} & =0.58 f_{\mathrm{b}}^{0.89}(a / b-1) \\
& =0.58 f_{\mathrm{b}}^{0.89} \frac{\epsilon_{\mathrm{bar}}}{1-\epsilon_{\mathrm{bar}}}, \quad \text { for } c=0
\end{aligned}
$$

or

$$
\begin{aligned}
Q_{\mathrm{b}} & =0.44 f_{\mathrm{b}}^{0.87}(a / b-1) \\
& =0.44 f_{\mathrm{b}}^{0.87} \frac{\epsilon_{\mathrm{bar}}}{1-\epsilon_{\mathrm{bar}}}, \quad \text { for } c=1
\end{aligned}
$$

(Kim et al. 2012). The bar mass fraction $f_{\mathrm{b}}$ is defined as $M_{\text {bar }} /\left(M_{\text {bar }}+M_{\text {bulge }}\right)$ where $M_{\text {bulge }}$ is measured by the bulge mass inside $r=10 \mathrm{kpc}$. The central density concentration $c=0$ denotes a homogeneous bar, whereas $c=1$ means a more concentrated bar. Comparing with the observational results of Comerón et al. (2010), Kim et al. (2012) suggested that bars in real galaxies are unlikely to be more concentrated than $c=1$ and might have a bar mass fraction of 0.25 to 0.5 . We also compare these analytic relations with our data: they are well represented by a homogeneous bar $(c=0)$ with $f_{\mathrm{b}}=0.4$ to 0.5 . We overplot the relation with $f_{\mathrm{b}}=0.4$ (gray line) and $f_{\mathrm{b}}=0.5$ (black line) for $c=0$ in Figure 13(a).

When it comes to the bar length measurements, $r_{\mathrm{Qb}}$ shows the strongest correlation with $r_{\mathrm{A} 2}$ (Figure 13(e)). The $Q_{\mathrm{T}}(r, \phi)$ map and Fourier analysis are quite consistent with each other in measuring the bar length, as reported in the previous study (Laurikainen et al. 2004a). We do not find any skewed distribution between earlyand late-type spirals in the bar length measurements (Figure 13(e)), differently shown in the bar strength measurements (Figure 13(b)). The outliers often come from confusions by the Fourier analysis misidentifying elongated bulges as bars. On the other hand, we find that even very long bars often show the maximum $Q_{\mathrm{b}}$ at the very inner regions of the bar.

In Figures 13(d) and (f), we find that the correlation between $r_{\epsilon}$ against $r_{\mathrm{Qb}}$ or $r_{\mathrm{A} 2}$ is not as tight as the correlation between $r_{\mathrm{Qb}}$ and $r_{\mathrm{A} 2}$. According to Díaz-García et al. (2016), $r_{\epsilon}$ best matches the bar length estimated by visual inspection. In our analysis, $r_{\mathrm{Qb}}$ and $r_{\mathrm{A} 2}$ are usually measured to be shorter by $20 \%$ than $r_{\epsilon}$.

\subsection{Dependence of Bar Properties on Galaxy Properties}

In Figure 14, we plot the bar strength and length as a function of the coded numerical revised Hubble stage $T$. We again used the three methods of measurement, the $Q_{\mathrm{T}}(r, \phi)$ map analysis, the ellipse fitting method, and the Fourier analysis. In each panel, we distinguish visually determined SBs, SABs, and SAs by Ann15 with successive offsets of +0.25 in the abscissa as red, blue, and gray dots, respectively. The lines indicate the mean values at the original bin. With $Q_{\mathrm{T}}(r, \phi)$ map analysis, we can measure the strength and length for all galaxies, whether or not the galaxy has a bar, as explained in $\S 3.1$. However, in other methods, we can estimate them only when the methods can detect bars. We note that the Fourier analysis rarely detects SABs (Paper I)

We show the bar strength versus the Hubble type in the upper row of Figure 14. The individual values are quite scattered, but the mean values show some dependence on the Hubble type. Even though the three bar strength measurements show some degree of correlation to each other (Figure 13), they do not show consistent tendencies as a function of the Hubble sequence. The mean values of $Q_{\mathrm{b}}$ increase toward the late-type spirals with high $T$ (Figure 14(a)), whereas the mean values of $A_{2}$ show the opposite trend, decreasing toward the late-type spirals (Figure 14(c)). The mean values of $\epsilon_{\mathrm{bar}}$ show no systematic dependence on the Hubble type (Figure 14(b)). The Hubble type dependence in $Q_{\mathrm{b}}$, increasing toward late-type spirals, has been previously reported (Laurikainen et al. 2002; Whyte et al. 2002; Buta et al. 2004, 2010b) and even the opposite tendencies between $Q_{\mathrm{b}}$ and $A_{2}$ also have been studied (Laurikainen et al. 2004a; Díaz-García et al. 2016).

SABs have lower bar strengths compared to SBs. The value of $Q_{\mathrm{b}}$ separates SBs from SABs or SAs most consistently compared to $A_{2}$ or $\epsilon_{\mathrm{bar}}$. The bar ellipticity $\epsilon_{\mathrm{bar}}$ is worst in separating SBs from SABs or SAs. However, the opposite tendencies for $\mathrm{T}$ do not differ in SABs and even in SAs.

In fact, we can expect these opposite distributions from Figure 13(b) where early-type spirals are skewed to larger $A_{2}$ and late-type spirals to larger $Q_{\mathrm{b}}$. This can be most likely explained by the bulge dilution: the underlying radial force generated by a bulge causes underestimation of $Q_{\mathrm{b}}$, especially in earlytype spirals (Block et al. 2001; Laurikainen \& Salo 2002; Laurikainen et al. 2004a; Buta et al. 2004; Elmegreen et al. 2007; Díaz-García et al. 2016). We test how much a bulge affects the estimation of the bar strength depending on the methods by using mock galaxies with different bulge-to-total ratios in $\S 6.1$.

Next, we display the relative bar length with respect to $R_{25}$ at the bottom row of Figure 14 . The pre- 

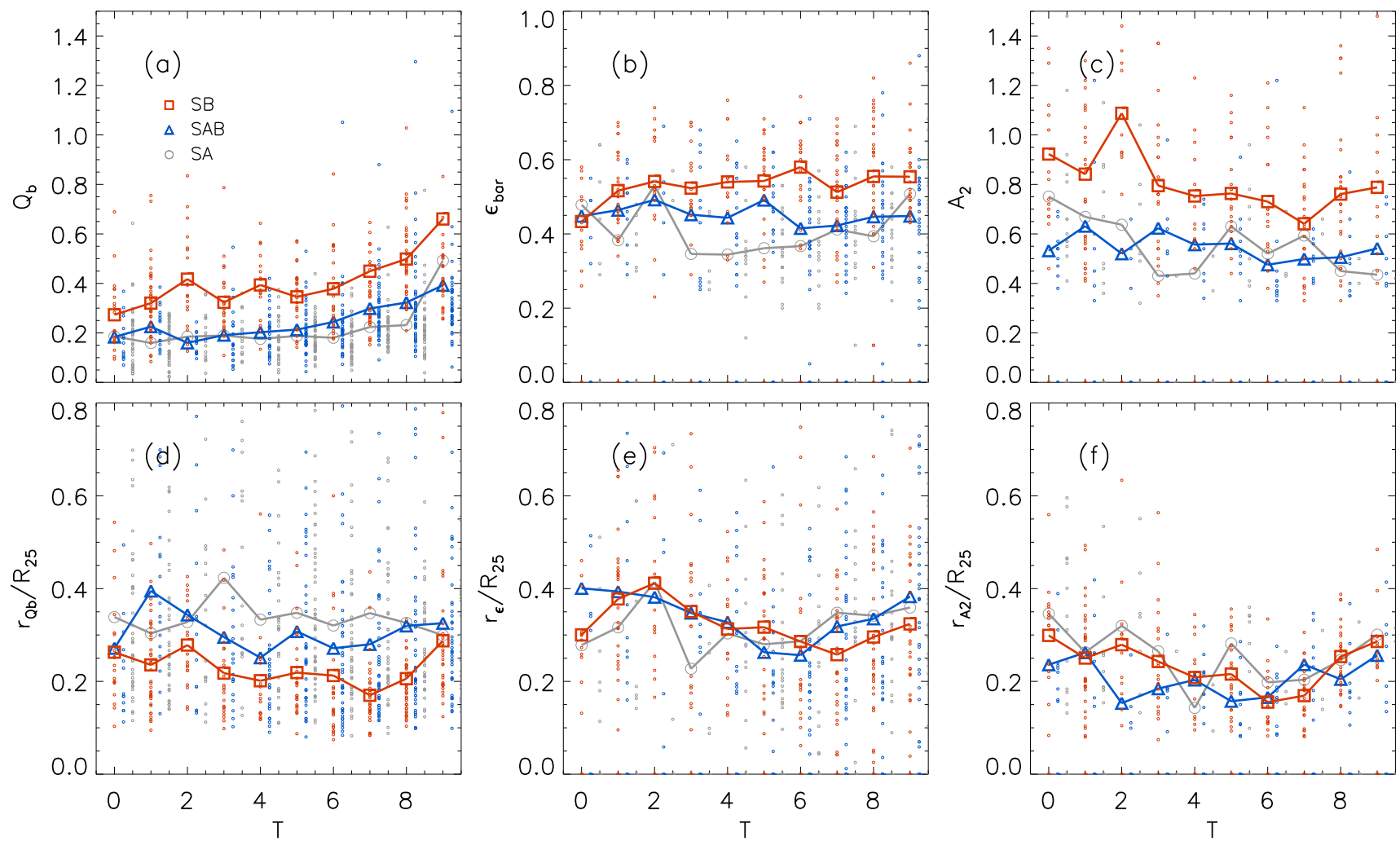

Figure 14. The bar strengths (upper row) and bar lengths normalized by $R_{25}$ (bottom row) as a function of the Hubble sequence. Three different measurements, $Q_{\mathrm{b}}$ (left), $\epsilon_{\mathrm{bar}}$ (middle), and $A_{2}$ (right), are used. The visually determined SBs, SABs, and SAs are displayed by red, blue, and gray colors with +0.25 offsets in the abscissa. The lines show mean values.

vious study shows that early-type spirals have longer bars in both absolute and relative lengths with respect to $R_{25}$ than late-type spirals (Elmegreen \& Elmegreen 1985; Martin 1995; Erwin 2005; Elmegreen et al. 2007; Menéndez-Delmestre et al. 2007; Aguerri et al. 2009). However, most of them used a small sample of galaxies or did not use the whole Hubble types. We find that early-type spirals have long bars, but the relative bar length increases from intermediate-type to late-type spirals as well. This result is consistent with the previous studies that considered the whole Hubble types, which show a bimodal distribution that the relative bar length increases towards both ends of the Hubble sequence with a minimum at $\mathrm{T}=5 \sim 6$ (Laurikainen et al. 2007; Díaz-García et al. 2016; Font et al. 2017, 2019). It means that the bar length does not decrease as much as the disk size decreases in late-type spirals. This tendency looks similar for all three different measurements, and both for SBs and SABs.

Lastly, we compare the bar length between SBs and SABs. Despite the general understanding that bars in SBs are highly contrasted, stronger, and longer compared to those in SABs (Elmegreen \& Elmegreen 1985; Ann \& Lee 1987; Elmegreen \& Elmegreen 1989; Ohta et al. 1990; Erwin 2005; Laurikainen et al. 2009;
Lee et al. 2012), we can not find in our analyses the evidence that bars in SBs are longer than those in SABs. The mean values of the relative bar length of SBs are not definitively greater than those of SABs in the same bin of Hubble type. In terms of $r_{\mathrm{Qb}}$, the mean bar lengths of SABs are even larger than those of SBs (Figure 14(d)). Accordingly, when we compare the results for $Q_{\mathrm{b}}$ and $r_{\mathrm{Qb}} / R_{25}$ in Figures 14(a) and (d), it is likely that the maximum point of the bar force ratio $r_{\mathrm{Qb}}$ is moving inwards as the bar is growing stronger. We find similar behaviour that $r_{\mathrm{Qb}}$ decreases as $Q_{\mathrm{b}}$ increases in a previous study (Laurikainen et al. 2002, see Figure 5) and also in the comparison with the simulation data by Seo et al. (2019), which is shown in Figure 19 of $\S 6.3$.

\section{DISCUSSION}

\subsection{Bulge Effect on Bar Properties}

Bars in early-type spirals have been believed to be longer and stronger than those in latetype spirals (Elmegreen \& Elmegreen 1985; Ann \& Lee 1987; Elmegreen \& Elmegreen 1989; Ohta et al. 1990; Elmegreen et al. 1996; Laurikainen et al. 2004a; Erwin 2005; Abraham \& Merrifield 2000; Laurikainen et al. 2009; Lee et al. 2012). Indeed, in terms of the Fourier amplitude, early-type spirals have stronger bars than 
late-type spirals (Figure 14(c)), which is consistent with previous studies (Ohta et al. 1990; Laurikainen et al. 2004a, 2009). However, in terms of the bar force ratio and bar ellipticity, it is hard to say that bars in earlytype spirals are stronger than those in late-type spirals (Figures 14(a) and (b)). This has also been reported in the literature (Laurikainen et al. 2002; Buta et al. 2004, 2010a; Laurikainen et al. 2004a; Díaz-García et al. 2016).

In order to understand the lower bar force ratio in early-type spirals, Buta et al. (2004) investigated several possible causes. They first took into account more spherical shapes of bulges by using two-dimensional decompositions for bulges, disks, and bars. The assumption for the bulge to be the same thin shape as the disk overestimates the radial force in the plane (Laurikainen \& Salo 2002; Buta et al. 2004). Secondly, they applied a constant ratio of $h_{r} / h_{z}=12$ for all Hubble type galaxies instead of the type dependent $h_{r} / h_{z}$ because high $h_{r} / h_{z}$ in late-type spirals enhances $Q_{\mathrm{b}}$. Thirdly, they tried to separate $Q_{\mathrm{b}}$ from the force ratio by spiral arms. Lastly, Buta et al. (2004) and Díaz-García et al. (2016) found that the dark halo correction lowers $Q_{\mathrm{b}}$ by $20 \sim 25 \%$ in $\mathrm{T}=7-10$. However, the dark halo could not fully explain the Hubble type dependence of $Q_{\mathrm{b}}$. The $Q_{\mathrm{b}}$ has still turned out to be lower in early-type spirals than in late-type spirals. We will discuss this more in $\S 6.2$.

On the other hand, it has been suggested that a bulge significantly dilutes $Q_{\mathrm{b}}$ in early-type spirals, for it is an important contributor to the radial force in the bar region (Block et al. 2001; Laurikainen \& Salo 2002; Buta et al. 2004; Laurikainen et al. 2004a; Elmegreen et al. 2007; Díaz-García et al. 2016). Laurikainen et al. (2004a) reported by investigating 180 disk galaxies that $Q_{\mathrm{b}}$ varies from 0.6 to 0.1 as the bulge-to-total ratio increases from 0 to 1 .

Here, we test the bulge effect on the value of the bar strength measured by all three different methods. We constructed various mock galaxies with the bulge-tototal ratio $(\mathrm{B} / \mathrm{T})$ of 0 to 0.8 . A bulge, a disk, and a bar with the flat or exponential profile were built as described in $\S 3.2$. In Figures 15(a)-(c), we display each measurement of the bar strength $Q_{\mathrm{b}}, \epsilon_{\mathrm{bar}}$, and $A_{2}$ as a function of $\mathrm{B} / \mathrm{T}$ for these mock galaxies. Red squares and blue triangles indicate flat and exponential barred galaxies, respectively. We find that not only $Q_{\mathrm{b}}$ but also $\epsilon_{\text {bar }}$ and $A_{2}$ largely depend on $\mathrm{B} / \mathrm{T}$. When $\mathrm{B} / \mathrm{T}$ increases from 0 to $0.8, Q_{\mathrm{b}}$ drops to less than half and $\epsilon_{\mathrm{bar}}$ reduces by around $30 \%$ (Figures 15(a)-(b)). In contrast, $A_{2}$ increases over by $50 \%$ (Figure 15(c)). This experiment explains the different Hubble type dependence of bar strength measured by different methods shown in Figure 14 , confirming the bulge dilution on $Q_{\mathrm{b}}$ suggested by the previous studies (Block et al. 2001; Laurikainen \& Salo 2002; Laurikainen et al. 2004a; Elmegreen et al. 2007; Díaz-García et al. 2016).

We emphasize that the bulge effect is as significant as the effect of the bar. It means that the measured bar strength for the same bar may decrease to half the value depending on the bulge that resides with the bar in the host galaxy. Besides, depending on the method to estimate the bar strength, the same bulge can decrease the bar strength $Q_{\mathrm{b}}$ and $\epsilon_{\mathrm{bar}}$ or increase the bar strength $A_{2}$.

In Figure 16, we plot the radial profiles of $\left\langle Q_{\mathrm{T}}\right\rangle, \epsilon$, and $A_{2}$ for all mock galaxies. The upper row shows flat barred galaxies, and the bottom row exponential barred galaxies. We indicate the $\mathrm{B} / \mathrm{T}$ ratio by the colors of lines: as the colors become lighter, $\mathrm{B} / \mathrm{T}$ increases from 0 to 0.8 . The cross symbols show the loci of the maximum bar strength. We confirm that when the color becomes lighter (B/T increases), $Q_{\mathrm{b}}$ and $\epsilon_{\mathrm{bar}}$ decrease, while $A_{2}$ increases. When it comes to the bar length, the locations of the maximum strength tend to move outward in most cases. These tendencies similarly appear in both flat and exponential barred galaxies.

To analyze the cause of the opposite trend between $Q_{\mathrm{b}}$ and $A_{2}$, we decomposed the profiles of $\left\langle Q_{\mathrm{T}}\right\rangle$ and $A_{2}$ into the components, $\left\langle F_{\mathrm{T}}\right\rangle,\left\langle F_{\mathrm{R}}\right\rangle, a_{2}$, and $a_{0}$ in Figure 17. We indicate the components in flat and exponential bars by red and blue colors, respectively. Lighter colors mean larger B/T as well. In Figures 17(a) and (b), we confirm that the profiles of tangential force $\left\langle F_{\mathrm{T}}\right\rangle$ and $a_{2}$ are hardly affected by the bulge ratio. They are the representative components of a bar. What we have to be cautious is that the radial force $\left\langle F_{\mathrm{R}}\right\rangle$ and $a_{0}$ are more strongly affected by the bulge than we have expected (Figures 17(c) and (d)). The higher B/T totally changes the profiles of $\left\langle F_{\mathrm{R}}\right\rangle$ and $a_{0}$, which results in the different bar strength and location of the maximum strength, i. e., bar length. We find that the opposite trends between $Q_{\mathrm{b}}$ and $A_{2}$ have been caused by the changing profiles of $\left\langle F_{\mathrm{R}}\right\rangle$ and $a_{0}$ as $\mathrm{B} / \mathrm{T}$ changes.

Therefore, we need to consider the bulge effect on the bar strength and length more carefully. Fortunately, in the case of the bar length, the bulge effect seems less significant compared to the bar strength (Figures 15(d)-(f)). However, we should note that when the B/T increases, the bar force ratio $Q_{\mathrm{b}}$ decreases but the bar length $r_{\mathrm{Qb}}$ becomes longer.

When we compare the types of bars, the exponential bars are more significantly affected by the bulge compared to the flat bars (Figures 15 and 16). In partic- 

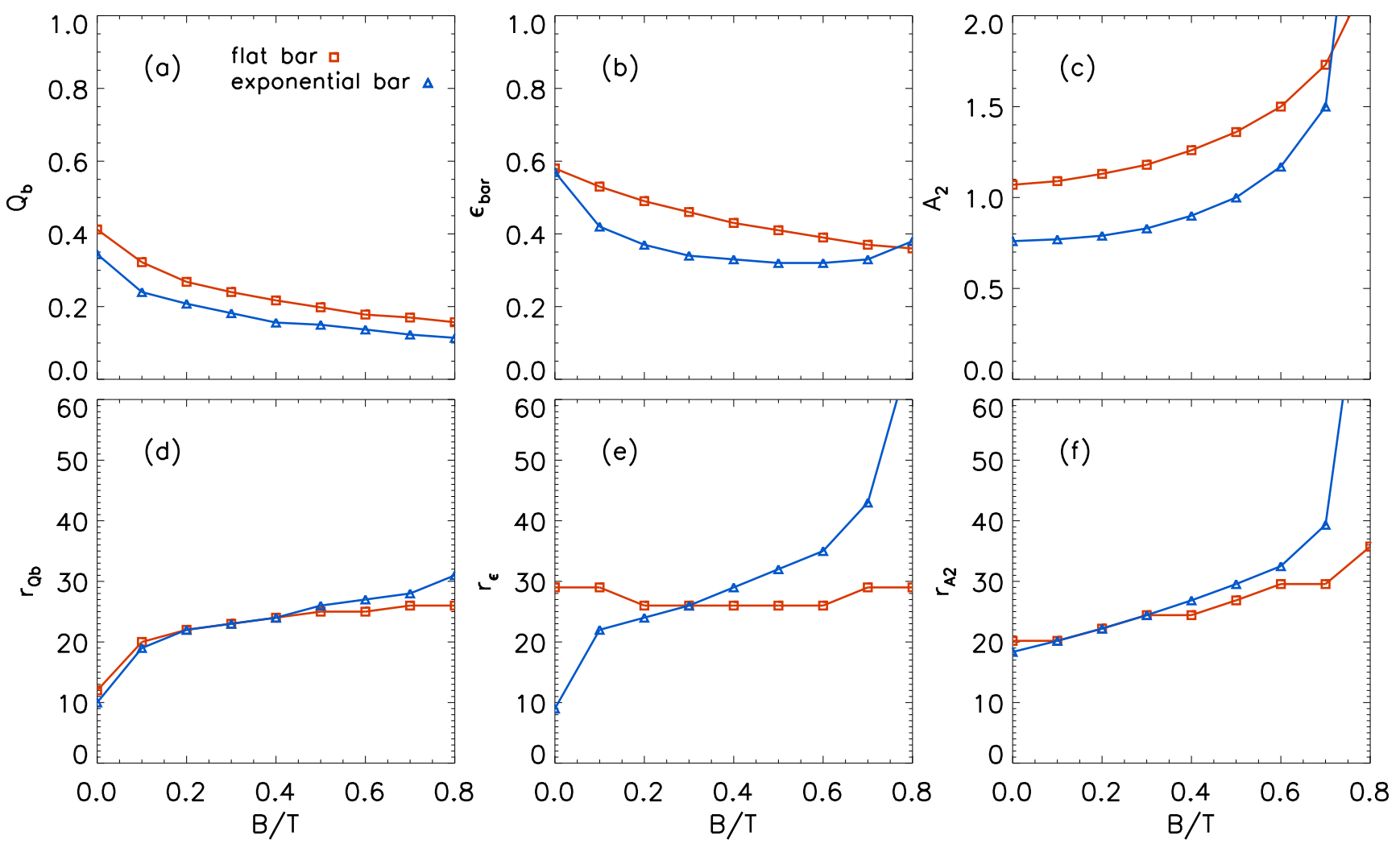

Figure 15. The bar strengths (upper row) and lengths (bottom row) as a function of the bulge-to-total ratio for mock galaxies with a bulge, a disk, and a bar. The bar strength and length are estimated by three different methods: $Q_{\mathrm{b}}$ and $r_{\mathrm{Qb}}$ from $Q_{\mathrm{T}}(r, \phi)$ map analysis $(l e f t), \epsilon_{\mathrm{bar}}$ and $r_{\epsilon}$ from ellipse fitting method (middle), and $A_{2}$ and $r_{A_{2}}$ from Fourier analysis (right). Red squares and blue triangles indicate flat barred galaxies and exponential barred galaxies, respectively.

ular, for the ellipse fitting, larger bulges in flat barred galaxies make the shape of a bar rounder and lower the ellipticity in the bar region (Figure 16(b)). Meanwhile, in exponential barred galaxies, ellipses are mainly determined by a bulge rather than a bar (Figure 16(e)). When the $\mathrm{B} / \mathrm{T}$ increases, $\epsilon_{\mathrm{bar}}$ decreases and $r_{\epsilon}$ is largely pushed outward.

\subsection{Variable Mass-to-light Ratio on Bar Force Ratio}

We have calculated the gravitational potential by assuming constant mass-to-light ratio $(\mathrm{M} / \mathrm{L})$ over the disk (Quillen et al. 1994). The stellar M/L is a vital parameter to translate photometry to dynamics (Bell \& de Jong 2001), and inevitably affected by the dark matter. We used $i$-band images to be less affected by the stellar population, but there are still variations in stellar $\mathrm{M} / \mathrm{L}$ within and among galaxies as large as a factor of 3 in $I$ band (Bell \& de Jong 2001). Bell \& de Jong (2001) showed that the stellar M/L has a strong correlation with the color of the integrated stellar population, and Buta et al. (2004) have suggested a way to consider the color-dependent $\mathrm{M} / \mathrm{L}$ in estimating the potential because of the radial stellar population variations, in particular, in late-type spirals. However, they did not choose to use the approach because of the impractical reason to adopt it to the full sample.

Here, we examine the dark matter effect on $Q_{\mathrm{b}}$ by adopting the color-dependent $\mathrm{M} / \mathrm{L}$ instead of the constant $\mathrm{M} / \mathrm{L}$. We have inferred the effective stellar mass at each pixel in $i$-band images with $(g-i)$ color maps, which gives the best agreement with those using optical/NIR color such as $(i-H)$ (Zibetti et al. 2009). We have constructed $(g-i)$ color maps for 120 galaxies, excluding objects that needed special reductions, among visually determined strongly barred galaxies in our sample. We adopt the relation between stellar $\mathrm{M} / \mathrm{L}$ and color $(g-i), \log _{10}(M / L)=-0.152+0.518 \times(g-i)$ (Bell et al. 2003).

In Figure 18, we display the force ratio from constant M/L (gray circle) against color-dependent M/L (red circle) as a function of the Hubble sequence. The mean $Q_{\mathrm{b}}$ from constant $\mathrm{M} / \mathrm{L}$ increases toward late-type spirals, although the values are lower than that for the whole sample as shown in Figure 14(a). The color-dependent $\mathrm{M} / \mathrm{L}$ tends to lower the mean $Q_{\mathrm{b}}$ over the whole Hubble sequence. As expected, the mean difference is minimal in $\mathrm{T}=0$ and $\mathrm{T}=1$, and becomes large, but within $20 \%$, in other Hubble types. The difference increases by 

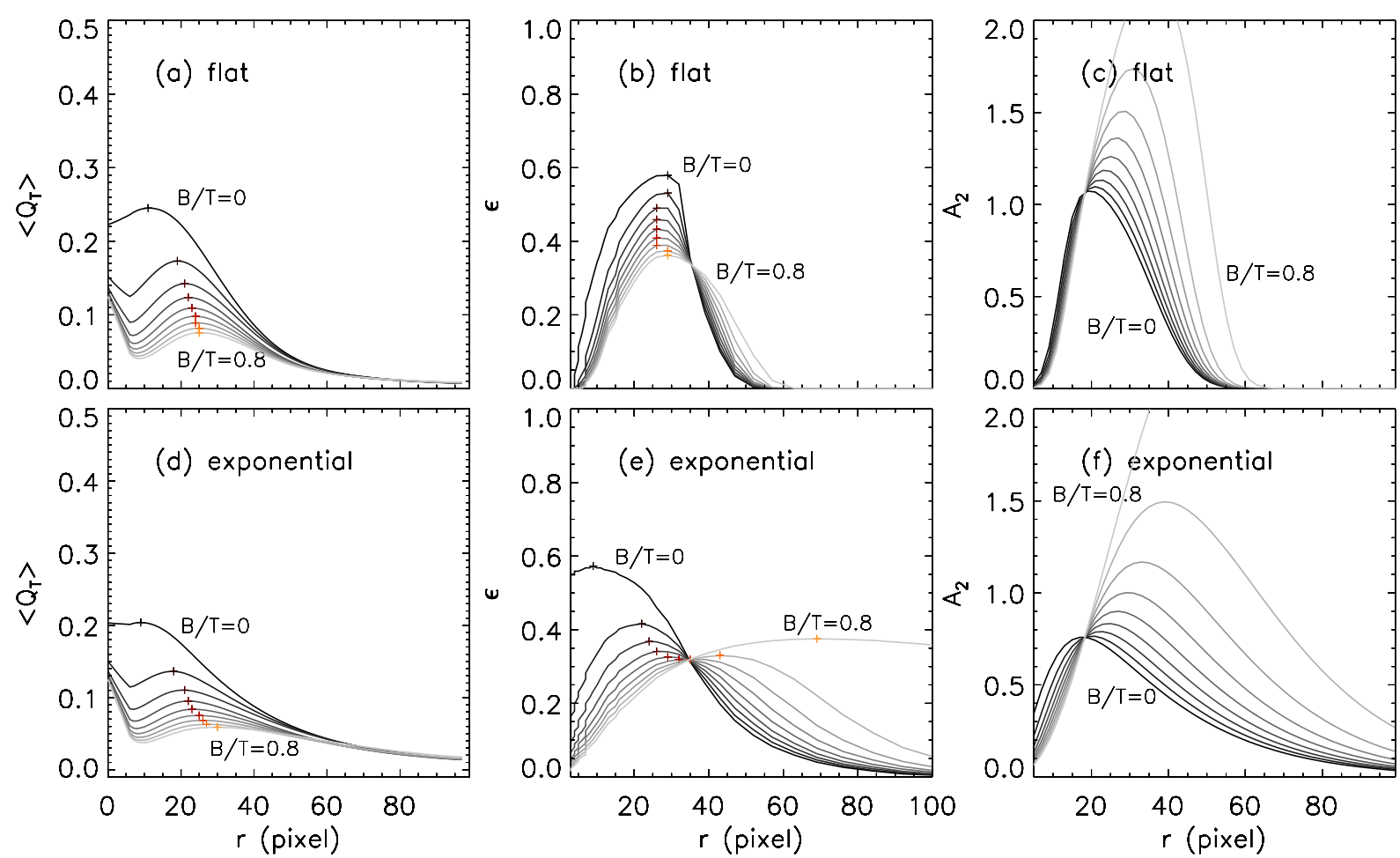

Figure 16. Radial profiles of $\left\langle Q_{\mathrm{T}}\right\rangle$ (left), $\epsilon$ (middle), and $A_{2}$ (right) for all individual mock galaxies. Lighter colors indicate larger $\mathrm{B} / \mathrm{T}$, from 0 to 0.8 . We plot the flat barred galaxies in the upper row and exponential galaxies in the bottom row. The cross symbols show the location of the maximum bar strength for each mock galaxy.
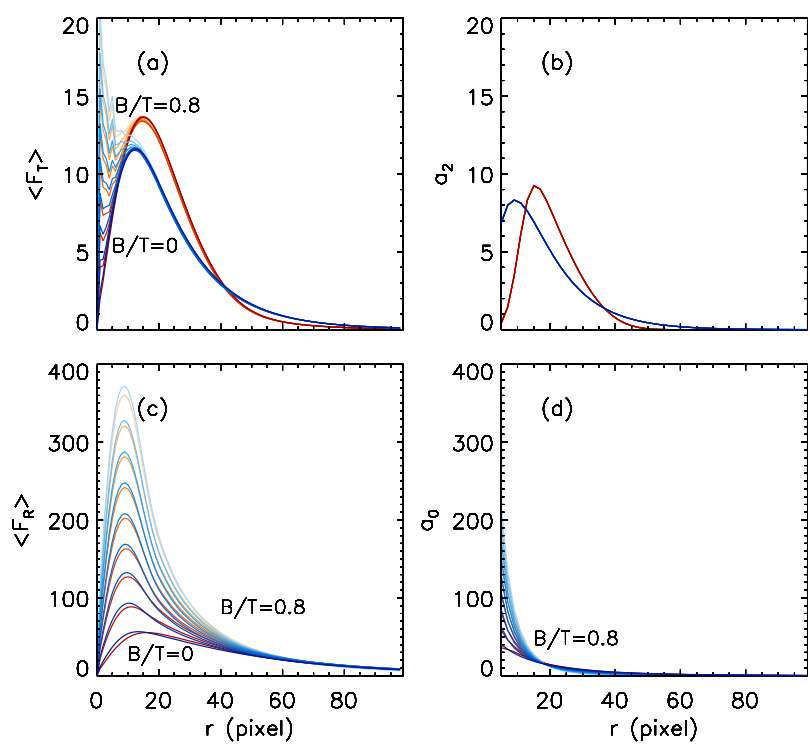

Figure 17. The components of $\left\langle Q_{\mathrm{T}}\right\rangle$ (left) and $A_{2}$ (right) for all individual mock galaxies. The upper row shows the radial profiles of $\left\langle F_{\mathrm{T}}\right\rangle$ and $a_{2}$, and the bottom row $\left\langle F_{\mathrm{R}}\right\rangle$ and $a_{0}$. Red and blue colors mean the components in flat and exponential barred galaxies. As colors become lighter, $\mathrm{B} / \mathrm{T}$ increases from 0 to 0.8 .

0.004 on average in the direction of lower $Q_{\mathrm{b}}$ as $\mathrm{T}$ increases by one. However, it does not change the general tendency of increasing $Q_{\mathrm{b}}$ toward late-type spirals. It is consistent with previous studies by Buta et al. (2004) and Díaz-García et al. (2016) who examined the dark matter effect on $Q_{\mathrm{b}}$ using the universal rotation curve models. Compared to the results by Díaz-García et al. (2016), the color-dependent M/L reflects the characteristics of individual galaxies such as the stellar population and dust lanes. Díaz-García et al. (2016) showed more systematic reduction of the mean $Q_{\mathrm{b}}$ by about $10-15 \%$ in $3 \leq T<5$ and $20-25 \%$ in $\mathrm{T}=7-10$.

\subsection{Comparison with a Numerical Simulation}

In $\S 4.1$, we introduced two types of the radial profile $\left\langle Q_{\mathrm{T}}\right\rangle(r)$ : one that has a maximum peak and the other a plateau on the radial profile (Figures 5 and 6 ). We label them type $\mathrm{M}$ or $\mathrm{P}$, respectively. We have classified our 884 sample galaxies into type M and P. Sometimes, a maximum peak looks like a plateau because the difference between the maximum and the minimum is minimal. However, as long as the profile has a maximum peak, we classified them as type M. In Table 2, we organize the fractions of type $\mathrm{M}$ and $\mathrm{P}$ for barred and nonbarred galaxies. We found the same fractions for type $\mathrm{M}(50 \%)$ and $\mathrm{P}(50 \%)$ from the whole sample. For barred galaxies, type M $(33 \%)$ is more common than type $\mathrm{P}(20 \%)$, while, in nonbarred galaxies, type $\mathrm{P}$ $(30 \%)$ is more frequent than type M $(17 \%)$. 


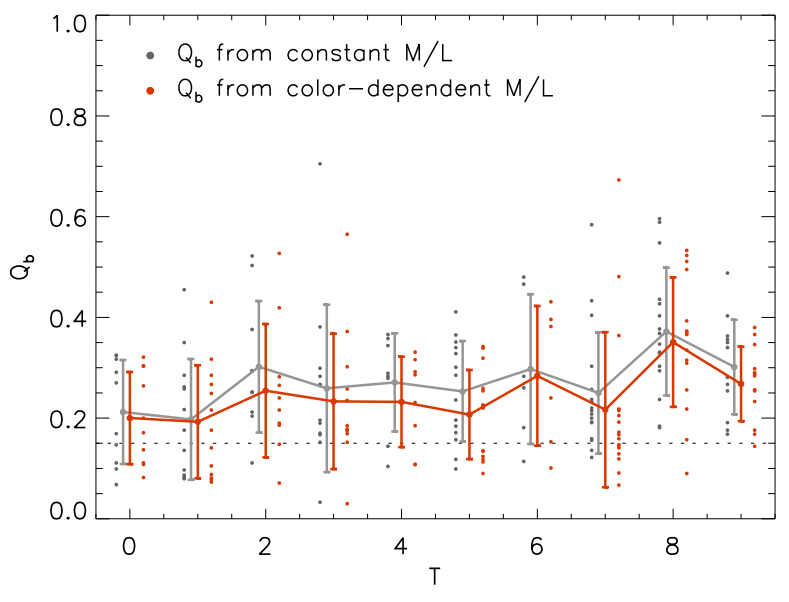

Figure 18. The force ratio $Q_{\mathrm{b}}$ as a function of the Hubble sequence. The red circles indicate $Q_{\mathrm{b}}$ obtained from adopting the constant $\mathrm{M} / \mathrm{L}$, and blue triangles mark corrected $Q_{\mathrm{b}}$ by applying the color-dependent $\mathrm{M} / \mathrm{L}$. The lines show the mean values.

Table 2. The Fractions of Type M and Type P Galaxy

\begin{tabular}{l|cc|c}
\hline bar/nonbar & type M & type P & total \\
\hline bar & $294(33 \%)$ & $174(20 \%)$ & $468(53 \%)$ \\
nonbar & $148(17 \%)$ & $268(30 \%)$ & $416(47 \%)$ \\
\hline total & $442(50 \%)$ & $442(50 \%)$ & $884(100 \%)$ \\
\hline
\end{tabular}

We were curious why the radial profiles appear to have two different types. In fact, we did not find any type $\mathrm{P}$ galaxies among our mock galaxies that have a flat or an exponential bar in Figures 16(a) and (d). Our visual inspection on the sample galaxies revealed that type $\mathrm{M}$ galaxies tend to have more developed bars along with other structures such as grand-design spiral arms or rings. On the other hand, type $\mathrm{P}$ galaxies rarely show prominent structures, except for bars. Thus, we suspect that the features in the radial profile might be related to the stage of galaxy evolution.

We have applied the $Q_{\mathrm{T}}(r, \phi)$ map analysis to the numerical simulation from Seo et al. (2019). They ran selfconsistent simulations of Milky Way-sized, isolated disk galaxies to explore the effect of a cold and warm disk in bar formation and evolution. The initial galaxy models are comprised of a stellar disk, a gaseous disk, a dark matter halo, and a central supermassive black hole. They considered relatively cold and warm disks with different velocity anisotropy parameters $f_{R} \equiv \sigma_{R}^{2} / \sigma_{z}^{2}=1.0$ and $f_{R}=1.44$, respectively. The total disk mass is fixed at $M_{\text {disk }}=5 \times 10^{10} M_{\odot}$, but the gas fraction varies from $0 \%$ to $10 \%$. The masses of a dark matter component and a supermassive black hole are $M_{\mathrm{DM}}=3.1 \times 10^{11} M_{\odot}$ and $M_{\mathrm{BH}}=4 \times 10^{6} M_{\odot}$, respectively.

Figure 19 shows the $Q_{\mathrm{T}}(r, \phi)$ map analysis for the warm-disk model with $5 \%$ gas fraction, which is considered to be the closest model to the Milky Way (Seo et al. 2019). The first column shows the stellar surface density in logarithmic scale in the $10 \mathrm{kpc}$ region. They are snapshots of the entire simulation from 0 Gyr to 5 Gyr. The simulation showed that the warm disk starts to form a weak bar at $t=0.4 \mathrm{Gyr}$, and the bar grows stronger until $t=2.0$ Gyr. The simulation often shows a spiral structure only without a bar for a short time at $t=0.8-0.9$ and at $t=1.4$ Gyr (Figures 19(d) and (f)) during the bar evolution (At 1.4 Gyr, our $Q_{\mathrm{T}}(r, \phi)$ analysis could not distinguish an elongated spiral arm from a bar). The second column presents $Q_{\mathrm{T}}(r, \phi)$ maps with the bar strength $Q_{\mathrm{b}}$ on the top right. The four thick slabs become longer, and the bar strengths increase until 2 Gyr. After 2 Gyr, the bar becomes a little weak up to 3 Gyr and grows again until 5 Gyr, the end of this simulation (Seo et al. 2019).

In the third column, we observe that the radial profile of $\left\langle Q_{\mathrm{T}}\right\rangle$ also grows with time. Until 0.3 Gyr (Figures 19(a) and (b)), the profile does not have any prominent structure except for the most central region. From 0.4 Gyr to 0.7 Gyr, we found a plateau outside the central region (Figure 19(c)). We classify the galaxy at this stage as type P. At this stage, we find weak bars in the stellar surface density images. The profile $\left\langle Q_{\mathrm{T}}\right\rangle$ grows to have a maximum peak from $0.8 \mathrm{Gyr}$ to the end of the simulation (Figures 19(d)-(g)). These stages are categorized as type M. In the stellar surface density images, we confirm that weak bars grow to strong bars, passing through a short stage of the nonbarred galaxy.

In this simulation, we observe that a disk grows roughly in sequence from a type $\mathrm{P}$ nonbar, a type $\mathrm{P}$ bar, a type $\mathrm{M}$ nonbar, to a type $\mathrm{M}$ bar. We may understand that the different features on the radial profiles might be related to the evolutionary stage of a bar. We speculate that a type $\mathrm{P}$ bar might be a young bar in the early stage, and a type $\mathrm{M}$ bar a more developed bar in the late stage of the bar evolution. This is consistent with our first expectation when we investigated the type $\mathrm{M}$ and $\mathrm{P}$ galaxies by a visual inspection. In addition, we can surmise that type $\mathrm{P}$ galaxies might be related to weakly barred galaxies and type $\mathrm{M}$ galaxies to strongly barred galaxies. We will discuss this more in $\S 6.4$.

In the fourth column, we display the azimuthal profile of $Q_{\mathrm{T}}$ at $r_{\mathrm{Qb}}$. The peaks grow in their heights with time, and the shapes also change. At 0.5 Gyr (Figure 19(c)), the shapes of four peaks are round, and the four peaks are equally spaced in $\phi$. However, as the bar grows, the 

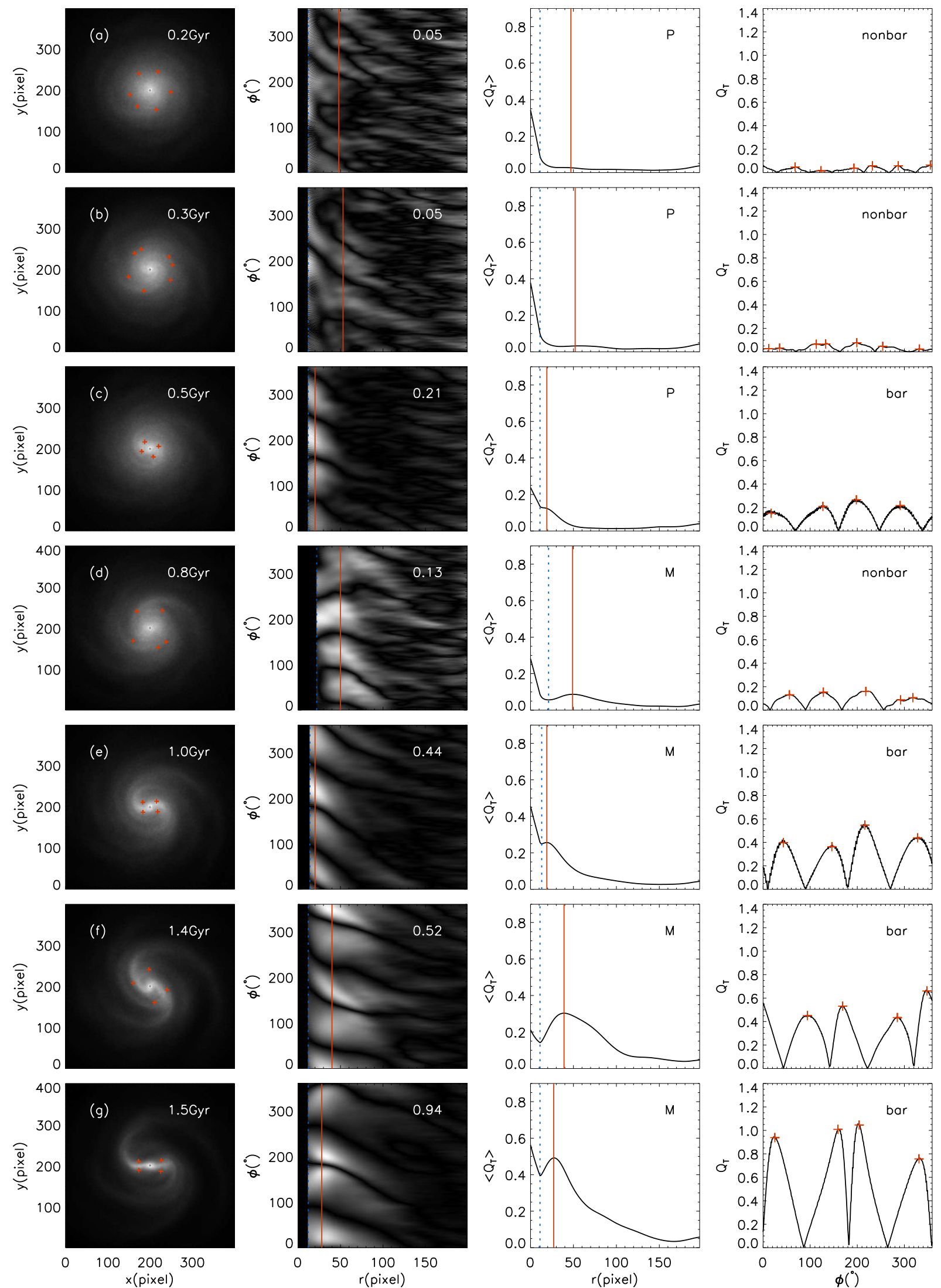

Figure 19. The $Q_{\mathrm{T}}(r, \phi)$ map analysis for the simulated galaxies that has a warm disk with $5 \%$ gas fraction from Seo et al. (2019). They are snapshots between 0 Gyr and 5 Gyr from top to bottom. Each snapshot is analyzed by the $Q_{\mathrm{T}}(r, \phi)$ map, radial profile, and azimuthal profile from left to right. We present the time scales, bar strengths $Q_{\mathrm{b}}, \mathrm{M}$ or $\mathrm{P}$ type, and bar or nonbar at the top right from left to right. 
shape of peaks becomes sharper, and the four peaks are coupled into two peaks (Figure 19(g)). In fact, we find similar coupled peaks in real sample galaxies, as shown in Figure 5 for NGC 4691. This may be a characteristic of a well-developed bar.

Lastly, we compare the type fraction of sample galaxies with that expected from the simulation data. In the simulation, the galaxy appears as a type $\mathrm{M}$ barred galaxy for $84 \%$ of its lifetime, while it stays at the type $\mathrm{P}$ for only $14 \%$ of its lifetime. However, we just find $33 \%$ type $\mathrm{M}$ barred galaxies in our observed sample and type $\mathrm{P}$ galaxies account for half of the whole sample. Therefore, considering the lifetime in the simulation, the fraction of type $\mathrm{M}$ barred galaxies in our sample is too small, whereas type $\mathrm{M}$ nonbarred galaxies and type $\mathrm{P}$ galaxies are too prevalent. Therefore, there seem to be some other factors that make barred galaxies not grow to type $\mathrm{M}$ or weaken to type $\mathrm{P}$ in real galaxies. We expect that the simulation study can describe real galaxies better if more accurate physical effects such as the effect of the bulge component is considered.

\subsection{Bar Classification and Hubble Sequence}

Paper I showed that the bar fraction as a function of the Hubble sequence depends on the method to detect barred galaxies. The bar fraction by the ellipse fitting method increases toward late-type spirals, while that by the Fourier analysis toward early-type spirals. We investigate the bar fractions obtained by our $Q_{\mathrm{T}}(r, \phi)$ map analysis as a function of the host galaxy properties such as the Hubble sequence, $g-r$, fracdeV, and $C_{\text {in }}$ (Figure 20). The fracdeV indicates the bulge-tototal ratio, which is estimated by the fraction of the light fit by the de Vaucouleurs profile (Masters et al. 2010, 2011; Lee et al. 2019). As the bulge dominates, the fracdeV value increases. The $C_{\text {in }}$ denotes the inverse light concentration, $C_{\text {in }} \equiv R_{50} / R_{90}$, where $R_{50}$ and $R_{90}$ are the Petrosian radii enclosing $50 \%$ and $90 \%$ of the total galaxy light, respectively (Petrosian 1976; Lee et al. 2012). We used the Hubble types and $g-r$ from Ann15, and other information from the SDSS database. We arrange all parameters in abscissa in the manners that early-type spirals are on the left, and late-type spirals on the right.

First, we find that the total bar fraction (gray solid lines) does not show any obvious or significant trend as functions of the properties of host galaxies. It looks similar to that of the total bar fraction determined by the visual inspection of Ann15 (Paper I, see Figure 6). Previous studies that have dealt with both SBs and SABs show a similar result (Knapen 1999; Eskridge et al. 2000; Li et al. 2017).
On the other hand, in the top row of Figure 20, when we divide SBs (red solid line) and SABs (blue dot-dashed line) based on the bar force ratio $Q_{\mathrm{b}}=0.25$, we find that SBs generally increase in late-type spirals, but SABs decrease in the same range. This differs from the visual inspection of Ann15: we reported that SBs are more frequent in early-type spirals, while SABs in intermediateor late-type spirals in Paper I. This is because a large bulge in early-type spirals dilutes the $Q_{\mathrm{b}}$ value, as discussed previously. Therefore, early-type barred galaxies are easier to be categorized as SABs compared to latetype barred galaxies.

Similarly, the bulge effect on the bar strength measurements discussed in $\S 6.1$ could also influence the bar fractions as a function of the Hubble sequence differently depending on the bar classification methods, ellipse fitting and Fourier analysis. In Figures 15(b) and (c), as $\mathrm{B} / \mathrm{T}$ increases, the bar ellipticity decreases, whereas the Fourier amplitude increases. It might affect detecting barred galaxies because of the bar criterion $\epsilon_{\text {bar }} \geq 0.25$ or $I_{2} / I_{0} \geq 0.3$ (Jogee et al. 2004; Laurikainen et al. 2002).

In the bottom row of Figure 20, we investigate the fractions of type $\mathrm{M}$ (red solid line) and type $\mathrm{P}$ (blue dot-dashed line) galaxies as functions of the host galaxy properties. We suspect that type $M$ barred galaxies are in a more advanced evolutionary state than are type $\mathrm{P}$ galaxies, as discussed in $§ 6.3$. The distribution of the fractions of type $\mathrm{M}$ and $\mathrm{P}$ resembles those of the fractions of $\mathrm{SB}$ and $\mathrm{SAB}$ by visual inspection, respectively. Type $M$ is more frequent in early-type, red, bulge-dominated, and more concentrated galaxies, while type $\mathrm{P}$ is more frequent in late-type, blue, diskdominated, and less concentrated galaxies.

We examine how the classification of type $\mathrm{M}$ and $\mathrm{P}$ is related to that of visually classified SB and SAB. In Figure 21, we compare our automated classification by $Q_{\mathrm{T}}(r, \phi)$ map analysis with the visual classification by Ann15. In this case, we use the whole sample of galaxies because the concordance sample rarely contains type $\mathrm{P}$ galaxies. Our method classifies galaxies into bar or nonbar and type $\mathrm{M}$ or $\mathrm{P}$ at the same time. The results of the classifications are summarized in Figure 21. We confirm that type $\mathrm{M}$ barred galaxies are most common in visually classified SB galaxies. Visually determined $\mathrm{SAs}$, on the contrary, have more type $\mathrm{P}$ galaxies than type $\mathrm{M}$ galaxies. However, we do not find the dominance of visually determined SABs in type $\mathrm{P}$ barred galaxies.

\section{SUMMARY}

We introduced a new method to classify barred galaxies by analyzing the transverse-to-radial force ratio map 

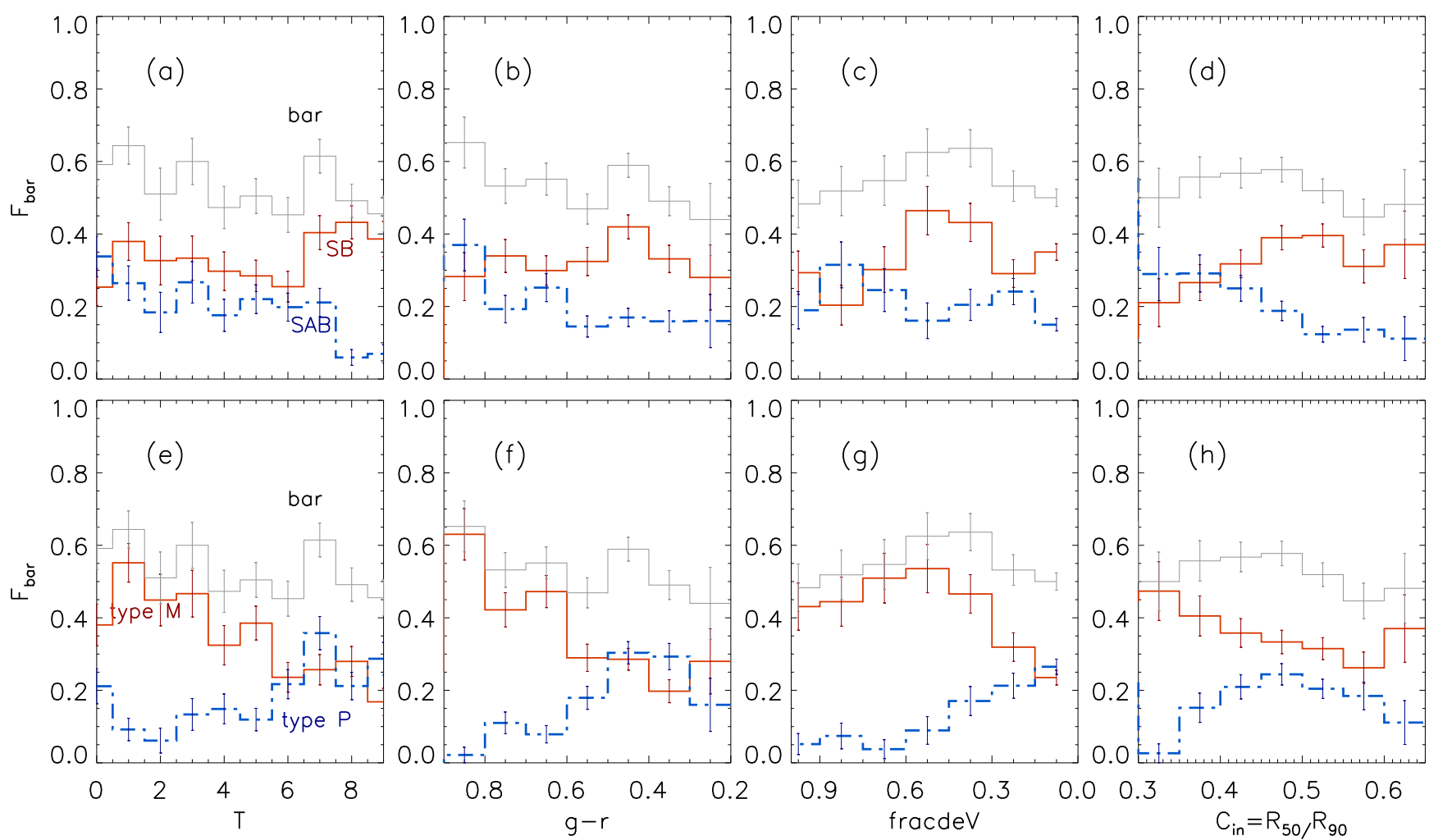

Figure 20. Dependence of the fraction of barred galaxies $F_{\mathrm{bar}}$ from the $Q_{\mathrm{T}}(r, \phi)$ map analysis on the host galaxy properties, the Hubble sequence, $g-r$, fracdeV and $C_{i n}$. The gray solid lines represent the total barred galaxies by the $Q_{\mathrm{T}}(r, \phi)$ map classification. In the top row, SBs (red solid line) and SABs (blue dot-dashed line) are distinguished by the criterion of $Q_{\mathrm{b}}=0.25$, while in the bottom row, type $\mathrm{M}$ (red solid line) and type $\mathrm{P}$ (blue dot-dashed line) are classified by the features in the radial profile: type $\mathrm{M}$ has a maximum peak, whereas type $\mathrm{P}$ has a plateau in the profile. We convert the abscissas of $g-r$ and fracdeV from right to left in order to arrange characteristics of early-type spirals on the left side in all panels.

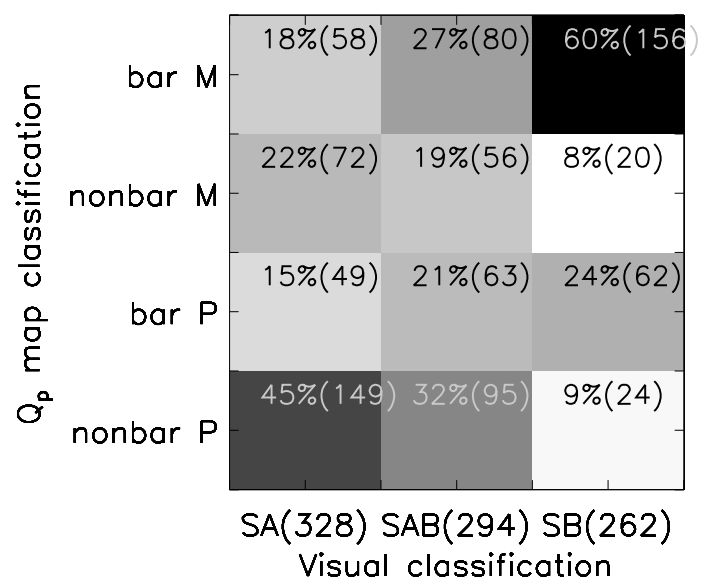

Figure 21. Comparison between classifications by analyzing $Q_{\mathrm{T}}(r, \phi)$ map and the visual inspection (Ann15). The $Q_{\mathrm{T}}(r, \phi)$ analysis provides the classification into bar or nonbar and type $\mathrm{M}$ or $\mathrm{P}$ (ordinate). The visual classification is shown in the abscissa. The numbers show how many galaxies classified by the visual inspection are classified into each classification by the $Q_{\mathrm{T}}$ map. through several steps: (1) We calculate the radial force and the transverse force by solving the Poisson equation with constant mass-to-light ratio. (2) We transform the ratio map from the Cartesian coordinates $Q_{\mathrm{T}}(i, j)$ to the polar coordinates $Q_{\mathrm{T}}(r, \phi)$ in order to simplify the butterfly pattern, the bar signature. (3) We investigate the radial profile of the force ratio, averaged over the azimuthal angle, $\left\langle Q_{\mathrm{T}}\right\rangle(r)$ and find the radius $r_{\mathrm{Qb}}$ where the mean force ratio shows a maximum peak or a plateau. (4) We investigate the azimuthal profile $Q_{\mathrm{T}}\left(r_{\mathrm{Qb}}, \phi\right)$ at $r_{\mathrm{Qb}}$ to see whether it has four peaks, corresponding to the four wings of the butterfly pattern. (5) We automatically classify galaxies as barred galaxies when they have four peaks at the azimuthal profile at $r_{\mathrm{Qb}}$ and a bar strength $Q_{\mathrm{b}}$ larger than 0.15.

In the following, we summarize the main results of this work.

1. This is a first application that utilizes the pattern of the transverse-to-radial force ratio map to classify barred galaxies. We successfully classified 
barred galaxies of $87 \%$ among visually determined barred galaxies by de Vaucouleurs et al. (1991) and Ann15 and distinguished nonbarred galaxies with an accuracy of $87 \%$. We apply this method to 884 spiral galaxies obtained from SDSS/DR7, which are volume-limited with $z<0.01$ and $M_{r}<-15.2$, and mildly-inclined with $i<60^{\circ}$, selected from 1876 parent sample galaxies. We obtain the bar fraction of $53 \%$, which is close to the bar fraction $(\sim 60 \%)$ by the classical visual inspection, including both SBs and SABs (Nilson 1973; Sandage \& Tammann 1987; de Vaucouleurs et al. 1991; Ann et al. 2015; Buta et al. 2015). Our method appears to be the most reliable way to select barred galaxies for large galaxy samples in the era of large galaxy surveys.

2. Our method provides measurements of the bar strength and the bar length in addition to the bar classification. In particular, we disentangle the bar strength from the spiral strength by analyzing the radial and azimuthal profile of the ratio map. We compare the bar strength $Q_{\mathrm{b}}$ and length $r_{\mathrm{Qb}}$ from our $Q_{\mathrm{T}}(r, \phi)$ map analysis with those from other measurements, the ellipse fitting and Fourier analysis. The bar strength and length measurements show relatively good correlations to each other. Notably, the $Q_{\mathrm{T}}(r, \phi)$ map and Fourier analysis are quite consistent with each other in measuring bar strength and length. However, we caution that they yield different tendencies for the Hubble sequence: as the Hubble sequence $T$ increases (later type), $Q_{\mathrm{b}}$ increases, whereas the normalized Fourier amplitude $A_{2}$ decreases. We show the bulge effect is significant in measuring the bar strength and length. In particular, the bulge causes underestimation of the bar strengths $Q_{\mathrm{b}}$ and $\epsilon_{\mathrm{bar}}$, but overestimation of the bar strength $A_{2}$ at the same time. When it comes to the bar length, the bulge makes overestimation in all three methods. We point out that we need to consider the bulge effect more carefully when studying the bar strength and the length than previously.

3. We find that visually determined SBs usually have stronger bar strength $Q_{\mathrm{b}}$ than visual SABs. Visual SABs show a similar distribution of $Q_{\mathrm{b}}$ with visual SAs rather than with visual SBs. We further subdivided barred galaxies as SBs if $Q_{\mathrm{b}} \geq 0.25$ and $\mathrm{SABs}$ if $0.15 \leq Q_{\mathrm{b}}<0.25$. However, this criterion tends to determine early-type barred galaxies as SABs easily but late-type barred galaxies as
SBs because bulges aid to underestimate $Q_{\mathrm{b}}$. It shows that the bulge effect on the bar strength measurements can influence the result of the bar classification. Besides, $39 \%$ of visually determined SABs have similar characteristics with nonbarred galaxies in manners that they do not have four peaks on the azimuthal profiles $Q_{\mathrm{T}}\left(r_{\mathrm{Qb}}, \phi\right)$, which supports the suggestion that $\mathrm{SAB}$ galaxies are the extension of SA galaxies (Abraham \& Merrifield 2000; Lee et al. 2019) or SAB galaxies have similar origin of the force ratio with SA galaxies (Laurikainen et al. 2004b).

4. We find two different types of the radial profile $\left\langle Q_{\mathrm{T}}\right\rangle(r)$. We classify our sample galaxies that have a maximum peak or a plateau on the radial profiles into type $\mathrm{M}$ or $\mathrm{P}$, respectively. We applied the same analysis to numerical simulations of bar evolution by Seo et al. (2019). The simulations show that galaxies change from type $\mathrm{P}$ to type $\mathrm{M}$ with time. We suspect that type $\mathrm{P}$ with a plateau might be in an early stage of the bar evolution, while type $M$ with a maximum peak is in a late stage of the bar evolution. Besides, the shape of four peaks at the azimuthal profile $Q_{\mathrm{T}}\left(r_{\mathrm{Qb}}, \phi\right)$ grows from the round shape with a similar width of $\phi$ to a sharp shape with two-to-two pair. It hints that we may estimate the evolutionary stage of bars by investigating the pattern of the ratio map.

We thank the anonymous referee for careful reading, helpful comments, and insightful guidance, which greatly improved this paper. We also thank WoongTae Kim for helpful discussion. We thank the BK21 Plus of the National Research Foundation of Korea (22A20130000179). YHL and MGP acknowledge support by the KASI under the R\&D program supervised by the Ministry of Science, ICT and Future Planning, by the National Research Foundation of Korea to the Center for Galaxy Evolution Research (No.2017R1A5A1070354), and by Basic Science Research Program through the National Research Foundation of Korea (NRF) funded by the Ministry of Education (No.2019R1I1A3A02062242). TK was supported by the Basic Science Research Program through the National Research Foundation of Korean (NRF) funded by the Ministry of Education (No.2019R1A6A3A01092024), and WYS by the grant (2017R1A4A1015178) of the National Research Foundation of Korea. 


\section{REFERENCES}

Abraham, R. G., \& Merrifield, M. R. 2000, AJ, 120, 2835

Aguerri, J. A. L. 1999, A\&A, 351, 43

Aguerri, J. A. L., Méndez-Abreu, J., \& Corsini, E. M. 2009, A\&A, 495, 491

Aguerri, J. A. L., Méndez-Abreu, J., Falcón-Barroso, J., et al. 2015, A\&A, 576, A102

Aguerri, J. A. L., Muñoz-Tuñón, C., Varela, A. M., \& Prieto, M. 2000, A\&A, 361, 841

Anderson, K. S. J., Baggett, S. M., \& Baggett, W. E. 2004, AJ, 127, 2085

Ann, H. B. 2001, Journal of Korean Astronomical Society, 34,261

Ann, H. B., \& Lee, S.-W. 1987, Journal of Korean Astronomical Society, 20, 49

Ann, H. B., \& Lee, H. M. 2000, Journal of Korean Astronomical Society, 33, 1

Ann, H. B., Seo, M., \& Ha, D. K. 2015, ApJS, 217, 27

Ann, H. B., \& Thakur, P. 2005, ApJ, 620, 197

Athanassoula, E. 1992, MNRAS, 259, 328

Athanassoula, E. 2002, ApJL, 569, L83

Athanassoula, E., Machado, R. E. G., \& Rodionov, S. A. 2013, MNRAS, 429, 1949

Athanassoula, E., \& Misiriotis, A. 2002, MNRAS, 330, 35

Athanassoula, E., Romero-Gómez, M., \& Masdemont, J. J. 2009, MNRAS, 394, 67

Athanassoula, E., Romero-Gómez, M., Bosma, A., et al. 2009, MNRAS, 400, 1706

Athanassoula, E., Romero-Gómez, M., Bosma, A., et al. 2010, MNRAS, 407, 1433

Athanassoula, E., Morin, S., Wozniak, H., et al. 1990, MNRAS, 245, 130

Athanassoula, E., Romero-Gómez, M., Bosma, A., et al. 2010, MNRAS, 407, 1433

Baggett, W. E., Baggett, S. M., \& Anderson, K. S. J. 1998, AJ, 116, 1626

Barazza, F. D., Jogee, S., \& Marinova, I. 2008, ApJ, 675, 1194

Baumgart, C. W., \& Peterson, C. J. 1986, PASP, 98, 56

Benedict, G. F., Higdon, J. L., Tollestrup, E. V., Hahn, J. M., \& Harvey, P. M. 1992, AJ, 103, 757

Bell, E. F., \& de Jong, R. S. 2001, ApJ, 550, 212

Bell, E. F., McIntosh, D. H., Katz, N., et al. 2003, ApJS, 149,289

Block, D. L., Puerari, I., Knapen, J. H., et al. 2001, A\&A, 375,761

Buta, R., \& Block, D. L. 2001, ApJ, 550, 243

Buta, R., Block, D. L., \& Knapen, J. H. 2003, AJ, 126, 1148

Buta, R. J., Corwin, H. G., \& Odewahn, S. C. 2007, The de Vaucouleurs Atlas of Galaxies
Buta, R. J., Knapen, J. H., Elmegreen, B. G., et al. 2009, AJ, 137, 4487

Buta, R., Laurikainen, E., \& Salo, H. 2004, AJ, 127, 279

Buta, R. J., Sheth, K., Regan, M., et al. 2010, ApJS, 190, 147

Buta, R., Laurikainen, E., Salo, H., et al. 2010, ApJ, 721, 259

Buta, R. J., Sheth, K., Athanassoula, E., et al. 2015, ApJS, 217, 32

Buta, R., Vasylyev, S., Salo, H., \& Laurikainen, E. 2005, AJ, 130, 506

Cheung, E., Athanassoula, E., Masters, K. L., et al. 2013, ApJ, 779, 162

Combes, F., \& Gerin, M. 1985, A\&A, 150, 327

Combes, F., \& Sanders, R. H. 1981, A\&A, 96, 164

Comerón, S., Knapen, J. H., Beckman, J. E., et al. 2010, MNRAS, 402, 2462

Comerón, S., Martínez-Valpuesta, I., Knapen, J. H., et al. 2009, ApJL, 706, L256

Consolandi, G. 2016, A\&A, 595, A67

Cuomo, V., Lopez Aguerri, J. A., Corsini, E. M., et al. 2019, A\&A, 632, A51

Davis, L. E., Cawson, M., Davies, R. L., \& Illingworth, G. 1985, AJ, 90, 169

de Grijs, R. 1998, MNRAS, 299, 595

de Vaucouleurs, G., de Vaucouleurs, A., Corwin, H. G., Jr., et al. 1991, S\&T, 82, 621

Díaz-García, S., Salo, H., Laurikainen, E., \& Herrera-Endoqui, M. 2016, A\&A, 587, A160

Ellison, S. L., Nair, P., Patton, D. R., et al. 2011, MNRAS, 416,2182

Elmegreen, B. G., \& Block, D. L. 1999, MNRAS, 303, 133

Elmegreen, B. G., \& Elmegreen, D. M. 1985, ApJ, 288, 438

Elmegreen, B. G., \& Elmegreen, D. M. 1989, ApJ, 342, 677

Elmegreen, B. G., Elmegreen, D. M., Chromey, F. R., Hasselbacher, D. A., \& Bissell, B. A. 1996, AJ, 111, 2233

Elmegreen, B. G., Elmegreen, D. M., Knapen, J. H., et al. 2007, ApJL, 670, L97

Erwin, P. 2005, MNRAS, 364, 283

Eskridge, P. B., Frogel, J. A., Pogge, R. W., et al. 2000, AJ, 119, 536

Flynn, C., \& Fuchs, B. 1994, MNRAS, 270,

Font, J., Beckman, J. E., James, P. A., et al. 2019, MNRAS, 482, 5362

Font, J., Beckman, J. E., Martínez-Valpuesta, I., et al. 2017, ApJ, 835, 279

Foyle, K., Courteau, S., \& Thacker, R. J. 2008, MNRAS, 386,1821

Frogel, J. A. 1985, ApJ, 298, 528 
Frogel, J. A., Persson, S. E., Aaronson, M., \& Matthews, K. 1978, ApJ, 220, 75

Garcia-Gómez, C., Athanassoula, E., Barberà, C., \& Bosma, A. 2017, A\&A, 601, A132

Grouchy, R. D., Buta, R. J., Salo, H., et al. 2010, AJ, 139, 2465

Heckman, T. M. 1980, A\&A, 88, 365

Hohl, F. 1972, Journal of Computational Physics, 9, 10

Hohl, F., \& Hockney, R. W. 1969, Journal of Computational Physics, 4, 306

Hoyle, B., Masters, K. L., Nichol, R. C., et al. 2011, MNRAS, 415, 3627

Huang, J. H., Gu, Q. S., Su, H. J., et al. 1996, A\&A, 313, 13

Hubble, E. P. 1926, ApJ, 64,

Jogee, S., Barazza, F. D., Rix, H.-W., et al. 2004, ApJL, 615, L105

Kalnajs, A. 1983, Internal Kinematics and Dynamics of Galaxies, 100, 87

Kim, T., Sheth, K., Gadotti, D. A., et al. 2015, ApJ, 799, 99

Kim, W.-T., Seo, W.-Y., \& Kim, Y. 2012, ApJ, 758, 14

Knapen, J. H. 1999, The Evolution of Galaxies on

Cosmological Timescales, 187, 72

Knapen, J. H., Pérez-Ramírez, D., \& Laine, S. 2002, MNRAS, 337, 808

Kormendy, J. 1977, ApJ, 217, 406

Kormendy, J. 1982, ApJ, 257, 75

Kormendy, J., \& Kennicutt, R. C. 2004, ARA\&A, 42, 603

Kuijken, K., \& Gilmore, G. 1989, MNRAS, 239, 571

Laurikainen, E., \& Salo, H. 2002, MNRAS, 337, 1118

Laurikainen, E., Salo, H., \& Buta, R. 2004, ApJ, 607, 103

Laurikainen, E., Salo, H., Buta, R., et al. 2004, MNRAS, 355,1251

Laurikainen, E., Salo, H., Buta, R., \& Knapen, J. H. 2009, ApJL, 692, L34

Laurikainen, E., Salo, H., Buta, R., \& Knapen, J. H. 2007, MNRAS, 381, 401

Laurikainen, E., Salo, H., \& Rautiainen, P. 2002, MNRAS, 331,880

Lee, G.-H., Park, C., Lee, M. G., \& Choi, Y.-Y. 2012, ApJ, 745,125

Lee, Y. H., Ann, H. B., \& Park, M.-G. 2019, ApJ, 872, 97

Li, Z.-Y., Ho, L. C., \& Barth, A. J. 2017, ApJ, 845, 87

Li, Z.-Y., \& Shen, J. 2015, ApJL, 815, L20

MacArthur, L. A., Courteau, S., \& Holtzman, J. A. 2003, ApJ, 582, 689

Marinova, I., \& Jogee, S. 2007, ApJ, 659, 1176

Marinova, I., Jogee, S., Heiderman, A., et al. 2009, ApJ, 698,1639
Marinova, I., Jogee, S., Trentham, N., et al. 2010, New Horizons in Astronomy: Frank N. Bash Symposium 2009, 432, 219

Marinova, I., Jogee, S., Weinzirl, T., et al. 2012, ApJ, 746, 136

Martin, P. 1995, AJ, 109, 2428

Martinet, L., \& Friedli, D. 1997, A\&A, 323, 363

Masters, K. L., Nichol, R. C., Hoyle, B., et al. 2011, MNRAS, 411, 2026

Masters, K. L., Nichol, R., Bamford, S., et al. 2010, MNRAS, 404, 792

Menéndez-Delmestre, K., Sheth, K., Schinnerer, E., et al. 2007, ApJ, 657, 790

Nair, P. B., \& Abraham, R. G. 2010, ApJL, 714, L260

Nilson, P. 1973, Acta Universitatis Upsaliensis. Nova Acta Regiae Societatis Scientiarum Upsaliensis - Uppsala Astronomiska Observatoriums Annaler, Uppsala: Astronomiska Observatorium, 1973,

Oh, S., Oh, K., \& Yi, S. K. 2012, ApJS, 198, 4

Ohta, K., Hamabe, M., \& Wakamatsu, K.-I. 1990, ApJ, 357,71

Pfenniger, D., \& Norman, C. 1990, ApJ, 363, 391

Peng, C. Y., Ho, L. C., Impey, C. D., \& Rix, H.-W. 2010, AJ, 139, 2097

Petrosian, V. 1976, ApJL, 209, L1

Prendergast, K. H. 1983, Internal Kinematics and Dynamics of Galaxies, 215

Puglielli, D., Widrow, L. M., \& Courteau, S. 2010, ApJ, 715,1152

Quillen, A. C., Frogel, J. A., \& Gonzalez, R. A. 1994, ApJ, 437, 162

Reese, A. S., Williams, T. B., Sellwood, J. A., Barnes, E. I., \& Powell, B. A. 2007, AJ, 133, 2846

Regan, M. W., \& Elmegreen, D. M. 1997, AJ, 114, 965

Regan, M. W., \& Teuben, P. J. 2004, ApJ, 600, 595

Roberts, W. W., Huntley, J. M., \& van Albada, G. D. 1979, ApJ, 233, 67

Rodgers, J. M., Kaur, C., Chen, Y.-G., et al. 2006, PhRvL, 97, 097801

Romero-Gómez, M., Athanassoula, E., Masdemont, J. J., et al. 2007, A\&A, 472, 63

Romero-Gómez, M., Masdemont, J. J., Athanassoula, E., et al. 2006, A\&A, 453, 39

Salo, H., Rautiainen, P., Buta, R., et al. 1999, AJ, 117, 792

Salo, H., Laurikainen, E., Buta, R., et al. 2010, ApJL, 715, L56

Sanders, R. H., \& Tubbs, A. D. 1980, ApJ, 235, 803

Sánchez-Menguiano, L., Pérez, I., Zurita, A., et al. 2015, MNRAS, 450, 2670 
Sandage, A., \& Tammann, G. A. 1987, Carnegie Institution of Washington Publication, Washington: Carnegie Institution, 1987, 2nd ed.,

Schwarz, M. P. 1981, ApJ, 247, 77

Schwarz, M. P. 1984, MNRAS, 209, 93

Seidel, M. K., Falcón-Barroso, J., Martínez-Valpuesta, I., et al. 2015, MNRAS, 451, 936

Sellwood, J. A., \& Wilkinson, A. 1993, Reports on Progress in Physics, 56, 173

Seo, W.-Y., Kim, W.-T., Kwak, S., et al. 2019, ApJ, 872, 5

Sheth, K., Elmegreen, D. M., Elmegreen, B. G., et al. 2008, ApJ, 675, 1141

Simmons, B. D., Melvin, T., Lintott, C., et al. 2014, MNRAS, 445, 3466
Skibba, R. A., Masters, K. L., Nichol, R. C., et al. 2012, MNRAS, 423, 1485

Terndrup, D. M., Davies, R. L., Frogel, J. A., Depoy, D. L., \& Wells, L. A. 1994, ApJ, 432, 518

van Albada, G. D., \& Roberts, W. W. 1981, ApJ, 246, 740

Yoshino, A., \& Yamauchi, C. 2015, MNRAS, 446, 3749

Whyte, L. F., Abraham, R. G., Merrifield, M. R., et al. 2002, MNRAS, 336, 1281

Wozniak, H., Friedli, D., Martinet, L., Martin, P., \& Bratschi, P. 1995, A\&AS, 111, 115

Zibetti, S., Charlot, S., \& Rix, H.-W. 2009, MNRAS, 400, 1181 